\title{
Fixed-point property of random quotients by plain words
}

\author{
HIROYASU IZEKI
}

\begin{abstract}
We show a fixed-point property of certain random groups for a wide class of CAT(0) spaces. The model of random groups under consideration is given as the set of presentations $(S, R)$, where $S$ is a generating set and the set of relations $R$ is a subset of the set of all plain words of the same length with suitably fixed density. Our main theorem says that groups obtained by such presentations have fixed-point property for all CAT(0) spaces having bounded singularities with high probability.
\end{abstract}

20F65; 58E20

\section{Introduction}

Let $Y$ be a metric space and $\operatorname{Isom}(Y)$ the group of isometries of $Y$. We say a group $\Gamma$ has fixed-point property for $Y$ if any isometric action of $\Gamma$ on $Y$ admits a fixed point; namely, for any homomorphism $\rho: \Gamma \longrightarrow \operatorname{Isom}(Y)$, there is a point $p \in Y$ such that $\rho(\gamma) p=p$ for all $\gamma \in \Gamma$. When we take a Hilbert space $\mathcal{H}$ as $Y$, according to theorems due to Delorme and Guichardet, the fixed-point property for $\mathcal{H}$ is equivalent to the Kazhdan's Property (T). Groups with Kazhdan's Property (T) have interesting and misterious feature as described in [1]. On the other hand, a group with the fixedpoint property for trees cannot be decomposed into an amalgamated free product as explained in [20].

Looking at these examples, one may suspect that having such a fixed-point property imposes a strong restriction on groups. Recent progress suggests that, however, groups with fixed-point property are distributed densely in a certain class of finitely generated groups as shown in [8], [21], [23], [9], [10], and [6]. In these papers, we see that certain random groups have strong fixed-point property: random groups in the triangle model ([23], [9]) and the graph model ([8], [21], [10], and [6]). Here, models of random groups are provided as certain sets of group presentations, and the phrase "random groups in the model admit fixed-point property" means that groups given by presentations in the model admit fixed-point property with high probability. In a slightly different direction, there is a result on so-called marked groups presented in 
[13], which claims that groups with fixed-point property is dense in the closure of the set of marked groups which are torsion-free, infinite, and non-elementary hyperbolic with respect to a certain topology. Also we should mention that the existence of a group with very strong fixed-point property is shown in [5] and [17]. Along the same lines as these results, our main theorems in the present paper say that groups with fixed-point property for certain metric spaces in fact form an extensive class among finitely presented groups; random quotients by plain words with certain densities admit strong fixed-point property as we explain below.

Let $m, \eta$ be natural numbers and $0<d<1$ a real number. Denote by $S$ a set consisting of $m$ letters and their inverses: $S=\left\{s_{1}, s_{1}^{-1}, \ldots, s_{m}, s_{m}^{-1}\right\}$. We call a sequence of letters belonging to $S$ a word, and the number of letters forming a word the length of the word. Thus, any word with length $\eta$ takes the form of $s_{i_{1}}^{\epsilon_{1}} s_{i_{2}}^{\epsilon_{2}} \ldots s_{i_{\eta}}^{\epsilon_{\eta}}$, where $\epsilon_{j}= \pm 1$ and $s_{i_{j}} \in S$. We denote by $W_{\eta}$ the set of words (plain words) of length $\eta$. We consider a group generated by $S$ with a presentation $P=(S, R)$, where the set of relations $R$ is a subset of $W_{\eta}$. We note that a relation $r \in R$ may be a reducible one, namely, $r$ may contain a sequence of the form $s_{i} s_{i}^{-1}$ or $s_{j}^{-1} s_{j}$. Fix a real number $c>1$ and set

$$
P(m, \eta, d)=\left\{P=(S, R) \mid R \subset W_{\eta} \text { and } c^{-1}(2 m)^{d \eta} \leq \# R \leq c(2 m)^{d \eta}\right\} .
$$

Let $\Gamma$ be the free group generated by $S: \Gamma=\left\langle s_{1}\right\rangle * \cdots *\left\langle s_{m}\right\rangle$. Then each element in $W_{\eta}$ determines an element in $\Gamma$ in an obvious way. Denote by $\Gamma_{P}$ the group given by a presentation $P=(S, R): \Gamma_{P}=\Gamma / \bar{R}$, where $\bar{R}$ is the normal closure of $R$ in $\Gamma$. It is an easy exercise to show that, for any finitely presented group $\Gamma$, there exists $m, \eta \in \mathbb{N}$ and $0<d<1$ such that $\Gamma=\Gamma_{P}$ for some $P \in P(m, \eta, d)$; that is, our model contains all finitely presented groups.

Let $Y$ be a $\operatorname{CAT}(0)$ space, a metric space with nonpositive curvature (see $\S 3$ for the definition). Complete, simply connected Riemannian manifolds with nonpositive sectional curvature, trees, and Hibert spaces are typical and important examples of CAT(0) spaces. When $\Gamma$ has fixed-point property for $Y$, we say $\Gamma$ has $F(Y)$ in what follows. Note that if $\Gamma$ is a finite group, then $\Gamma$ has fixed-point property for any CAT(0) space. In fact, an orbit of any isometric action of $\Gamma$ on $Y$ consists of finite points, and hence, in $\operatorname{CAT}(0)$ space $Y$, we can find the barycenter of the orbit that must be invariant under the action of $\Gamma$. (See Proposition 3.2 for the definition and the existence of the barycenter.) Thus, in what follows, our interest is in an infinite group $\Gamma$ with fixed-point property for CAT(0) spaces.

If we take $d$ in $P(m, \eta, d)$ larger, then producing homomorphism from $\Gamma_{P}$ for $P \in$ $P(m, \eta, d)$ into $\operatorname{Isom}(Y)$ becomes more difficult, which means that isometric actions of 
$\Gamma_{P}$ on $Y$ must carry strong restriction, in general. This leads us to a naive consideration that the larger $d$ becomes, the more we can expect $\Gamma_{P}$ for $P \in P(m, \eta, d)$ to have fixedpoint property. On the other hand, if we take $d$ too large, then $R$ becomes large; it may force $\Gamma_{P}$ to be a finite group, which is the case we want to avoid. Our main theorems tell us that the truth is exactly as this consideration describes, and there does exist $d$ which provides infinite groups with fixed-point property for a certain class of CAT(0) spaces with high probability. For Hilbert spaces, we will show the following theorem.

Theorem 1.1 Let $\mathcal{H}$ be a Hilbert space. For $1 / 3<d<1-\frac{1}{2} \log _{2 m}(8 m-4)$,

$$
\lim _{\eta \rightarrow \infty} \frac{\#\left\{P \in P(m, \eta, d) \mid \Gamma_{P} \text { is an infinite group having } F(\mathcal{H})\right\}}{\# P(m, \eta, d)}=1
$$

holds.

If we only allow irreducible words to be relations in the definition of $P(m, \eta, d)$, we obtain the density model of Gromov. One can expect a result for the density model similar to Theorem 1.1, and an idea of the proof, a reduction to Zuk's theorem ([23, Theorem 4]), is suggested in [18, I.3.g $]^{1}$.

In order to state our result for general $\mathrm{CAT}(0)$ spaces, we need to introduce an invariant of $\mathrm{CAT}(0)$ spaces defined in [11, Definition 6.1]: Let $\mu$ be a measure on a $\mathrm{CAT}(0)$ space $Y$, whose support supp $\mu$ consists of finite points. Denote by $\bar{\mu}$ the barycenter of $\mu$ (see Proposition 3.2), and let

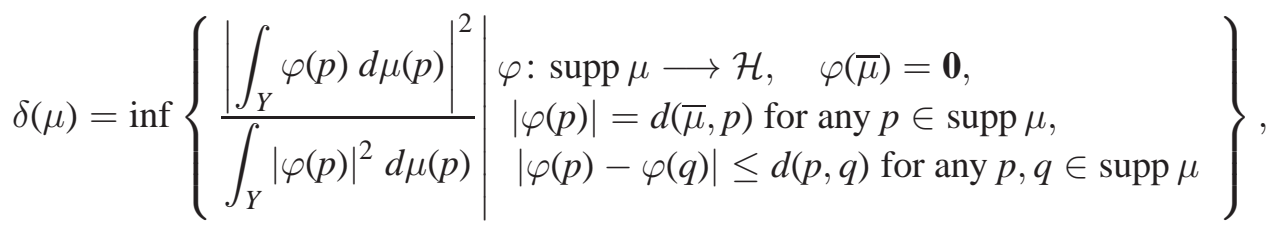

where $\mathcal{H}$ is a Hilbert space, and $\mathbf{0}$ denotes its origin. Then $\delta(Y)$ is defined to be

$$
\delta(Y)=\sup \{\delta(\mu) \mid \mu \text { is a measure on } Y \text { with \#supp } \mu<\infty\} \text {. }
$$

This invariant $\delta(Y)$ measures the singularity of $Y$, and obviously it takes values in $[0,1]$. If $\delta(Y)=0$, then $Y$ is considered to be almost regular; CAT(0) Riemannian manifolds and trees satisfy $\delta(Y)=0$. For general CAT(0) spaces we will show:

Theorem 1.2 Take $0 \leq \delta<1$ and an integer $k$ so that $k>1 /(1-\delta)$. Let $\mathcal{Y}_{\delta}$ be the class of CAT(0) spaces with $\delta(Y) \leq \delta$. Then, for $k /(2 k+1)<d<1-\frac{1}{2} \log _{2 m}(8 m-4)$,

$$
\lim _{\eta \rightarrow \infty} \frac{\#\left\{P \in P(m, \eta, d) \mid \Gamma_{P} \text { is an infinite group having } F(Y) \text { for all } Y \in \mathcal{Y}_{\delta}\right\}}{\# P(m, \eta, d)}=1
$$

\footnotetext{
${ }^{1}$ After the present paper was submitted, a complete proof was given by Marcin Kotowski and Michał Kotowski [16].
} 
holds.

A part of the theorems above is due to Ollivier; Ollivier [19] proved that, by choosing $d<1-\frac{1}{2} \log _{2 m}(8 m-4)$,

$$
\lim _{\eta \rightarrow \infty} \frac{\#\left\{P \in P(m, \eta, d) \mid \Gamma_{P} \text { is non-elementary hyperbolic }\right\}}{\# P(m, \eta, d)}=1,
$$

where $\Gamma$ is non-elementary hyperbolic means that $\Gamma$ is infinite hyperbolic group (in the sense of Gromov) which does not contain $\mathbb{Z}$ as a finite index subgroup. Since $\frac{1}{2} \log _{2 m}(8 m-4) \rightarrow 1 / 2$ as $m \rightarrow \infty$, by taking $m$ sufficiently large, Theorem 1.2 tells us that, for any $0 \leq \delta<1$, we can choose $d$ so that infinite hyperbolic groups with $F\left(\mathcal{Y}_{\delta}\right)$ form an extensive class among the groups coming from $P(m, \eta, d)$. Here a group $\Gamma$ has $F\left(\mathcal{Y}_{\delta}\right)$ means that $\Gamma$ has $F(Y)$ for all $Y \in \mathcal{Y}_{\delta}$. We note that, in [6], it is shown that any $\operatorname{CAT}(0)$ cube complex $Y$ satisfies $\delta(Y) \leq 1 / 2$. Therefore $\mathcal{Y}_{1 / 2}$ contains all CAT(0) Riemannian manifolds and CAT(0) cube complexes. On the other hand, there does exist $\mathrm{CAT}(0)$ space $Y$ with $\delta(Y)=1$ ([14]).

This paper is organized as follows. In $\S 2$, we introduce a finite graph associated to each element of $P(m, \eta, d)$ and state Proposition 2.1, which concerns with the eigenvalue of random graphs associated to $P(m, \eta, d)$. In $\S 3$, we introduce an energy of equivariant maps from $\Gamma$ to $Y$, and give a criterion for an action of $\Gamma$ on $Y$ to have a fixed point in terms of the ratio of certain energies, which is a modification of [10, Lemma 2.7(2)]. We also state Proposition 3.7, which connects the ratio of energies and the eigenvalue of the graph introduced in $\S 2$. In $\S 4$, we give the proofs of Theorem 1.1 and 1.2, assuming Propositions 2.1 and 3.7. After a brief review of some facts in probability theory in $\S 5$, we give the proofs of Propositions 2.1 and 3.7 in $\S 6$. As a matter of fact, our proofs give results on random quotients of any group, which will be explained in $\S 7$.

Acknowledgement. The author would like to thank Shin Nayatani and Takefumi Kondo for their interest in this work. The author is also grateful to Yann Ollivier for his valuable comments on the first manuscript of this paper.

\section{A graph associated to a presentation}

Fix a natural number $k$. For each natural number $\eta \geq k$, we take $l$ so that $l$ is the largest integer satisfying $\eta \equiv l(\bmod 2)$ and $l \leq \eta /(2 k+1)$. We set $2 n=\eta-l$. Therefore we have

$$
\begin{aligned}
(2 k+1) l & \leq \eta=(2 n+l) \leq(2 k+1) l+2(2 k+1), \\
k l & \leq n \leq k l+(2 k+1) .
\end{aligned}
$$


Note that $\eta \rightarrow \infty$ implies both $l \rightarrow \infty$ and $n \rightarrow \infty$.

Let $0<d<1$ and $P \in P(m, \eta, d)$. We associate a finite graph $G_{P}$ to $P=(S, R)$ as follows: the vertex set $V$ of $G_{P}$ is identified with $W_{n}$ and a pair $\left\{v_{1}, v_{2}\right\} \in V \times V$ is joined by an edge if there is a word $v_{1} u v_{2}^{-1} \in R \subset W_{\eta}$, where $u \in W_{l}$ and $v^{-1}$ denotes the inverse word $s_{i_{n}}^{-\epsilon_{n}} \ldots s_{i_{1}}^{-\epsilon_{1}}$ of $v=s_{i_{1}}^{\epsilon_{1}} \ldots s_{i_{n}}^{\epsilon_{n}}, \epsilon_{j}= \pm 1$. We denote the set of (unoriented) edges of $G_{P}$ by $E_{P}$. Thus $G_{P}=\left(V, E_{P}\right)$, where

$$
\left\{\begin{array}{l}
V=W_{n}, \\
E_{P}=\left\{\left\{v_{1}, v_{2}\right\} \mid v_{1} u v_{2}^{-1} \in R \text { for some } u \in W_{l}\right\} .
\end{array}\right.
$$

If there are two words $u, u^{\prime} \in W_{l}$ such that $r=v_{1} u v_{2}^{-1}, r^{\prime}=v_{1} u^{\prime} v_{2}^{-1} \in R$, then $r$ and $r^{\prime}$ define distinct edges with the same endpoints $v_{1}, v_{2} \in V$; thus $G_{P}$ may have multiple edges. Also $G_{P}$ may have self loops, since $r=v u v^{-1}$ may appear in $R$. Let $\nu$ be a measure on $V \times V$ defined by

$$
\nu\left(v_{1}, v_{2}\right)=\frac{\#\left\{u \in W_{l} \mid v_{1} u v_{2}^{-1} \in R\right\}+\#\left\{u^{\prime} \in W_{l} \mid v_{2} u^{\prime} v_{1}^{-1} \in R\right\}}{2 \# R} .
$$

By definition, $\nu$ is a symmetric probability measure on $V \times V$ and can be regarded as a probability measure on $E_{P}$. By a slight abuse of notation, we denote by $\nu$ a probability measure (or simply a funtion) on $V$ defined as

$$
\nu(v)=\sum_{v^{\prime}} \nu\left(v, v^{\prime}\right)=\frac{\#\left\{u \in W_{n+l} \mid v u \in R\right\}+\#\left\{u^{\prime} \in W_{n+l} \mid u^{\prime} v^{-1} \in R\right\}}{2 \# R} .
$$

Note that

$$
\nu(v)=\frac{\operatorname{deg}(v)}{2 \# R}=\frac{\operatorname{deg}(v)}{2 \# E_{P}},
$$

where $\operatorname{deg}(v)$ is the degree of $v$, which is defined to be the cardinality (counted with multiplicity) of edges having $v$ as one of the endpoints. Since a self loop comes from a relation of the form $v u v^{-1}$, it is counted twice in the definition of our degree. For real valued functions $f, g$ defined on $V$, their inner product is defined by

$$
(f, g)_{L^{2}}=\sum_{v \in V} \nu(v) f(v) g(v) .
$$

The Laplacian $\Delta_{P}$ of $G_{P}$ is defined by

$$
\Delta_{P} f(v)=f(v)-\sum_{v^{\prime}} \frac{\nu\left(v, v^{\prime}\right)}{\nu(v)} f\left(v^{\prime}\right),
$$

for a funtion $f: V \longrightarrow \mathbb{R}$ on $V$. It is easy to see that $\Delta_{P}$ is a symmetric and nonnegative operator with respect to the inner product $(\cdot, \cdot)_{L^{2}}$ defined above. The eigenvalues of $\Delta_{P}$ are distributed in the interval $[0,2]$, and it is clear that constant functions are 
eigenfunctions corresponding to the smallest eigenvalue 0 . We denote by $\lambda_{1}\left(G_{P}\right)$ the second smallest eigenvalue of $\Delta_{P}$ counted with multiplicity, which is positive if and only if $G_{P}$ is connected. The Rayleigh quotient $R Q(f)$ of $f: V \longrightarrow \mathbb{R}$ is defined as

$$
R Q(f)=\frac{\|d f\|_{L^{2}}^{2}}{F(f)}
$$

where

$$
\left\{\begin{array}{l}
\left.\|d f\|_{L^{2}}^{2}=\frac{1}{2} \sum_{v \in V} \sum_{v^{\prime} \in V} \nu\left(v, v^{\prime}\right) \mid f(v)-f\left(v^{\prime}\right)\right)\left.\right|^{2} \\
F(f)=\frac{1}{2} \sum_{v, v^{\prime} \in V} \nu(v) \nu\left(v^{\prime}\right)\left|f(v)-f\left(v^{\prime}\right)\right|^{2} .
\end{array}\right.
$$

Easy computations show that $F(f)$ is equal to the $L^{2}$-norm of the component of $f$ which is perpendicular to constant functions and that $\|d f\|_{L^{2}}^{2}=\left(f, \Delta_{P} f\right)_{L^{2}}$. Therefore we obtain

$$
\lambda_{1}\left(G_{P}\right)=\inf \{R Q(f) \mid f \text { is a nonconstant function on } V\} .
$$

In $\S 6.2$, We will show the following proposition on the eigenvalue of the Laplacian of $G_{P}$.

Proposition 2.1 Fix a natural number $k$. If $k /(2 k+1)<d$, then, for any $\varepsilon>0$,

$$
\lim _{\eta \rightarrow \infty} \frac{\#\left\{P \in P(m, \eta, d) \mid \lambda_{1}\left(G_{P}\right)>1-\varepsilon\right\}}{\# P(m, \eta, d)}=1
$$

holds.

\section{Energy of equivariant maps and fixed points}

In this section, we review and improve some results obtained in [9] and [10], which concern the energy of equivariant maps from a finitely generated group to a $\mathrm{CAT}(0)$ space. A complete metric space $Y$ is called CAT(0) space if any geodesic triangle is thinner than that in the Euclidean plane in the following sense.

Let $Y=\left(Y, d_{Y}\right)$ be a complete metric space and $p, q \in Y$. A geodesic joining $p$ to $q$ is an isometric embedding $c$ of a closed interval $[0, l]$ into $Y$ such that $c(0)=p$ and $c(l)=q$, where $l=d_{Y}(p, q)$. If any pair of points in $Y$ are joined by a geodesic, then $Y$ is called a geodesic space. Consider a triangle in a geodesic space $Y$ with vertices $p_{1}, p_{2}, p_{3} \in Y$ and three geodesic segments $p_{1} p_{2}, p_{2} p_{3}, p_{3} p_{1}$ joining them. We denote this triangle by $\Delta\left(p_{1}, p_{2}, p_{3}\right)$ and call such a triangle a geodesic triangle. Note that we can take a triangle $\Delta\left(\overline{p_{1}}, \overline{p_{2}}, \overline{p_{3}}\right)$ in $\mathbb{R}^{2}$ so that $d_{Y}\left(p_{i}, p_{j}\right)=d_{\mathbb{R}^{2}}\left(\overline{p_{i}}, \overline{p_{j}}\right)$, since the side lengths of $\Delta\left(p_{1}, p_{2}, p_{3}\right)$ satisfy the triangle inequality. We call $\Delta\left(\overline{p_{1}}, \overline{p_{2}}, \overline{p_{3}}\right)$ a 
comparison triangle for $\Delta\left(p_{1}, p_{2}, p_{3}\right)$. And we call a point $\bar{q} \in \overline{p_{i} p_{j}}$ a comparison point for $q \in p_{i} p_{j}$ if $d_{Y}\left(p_{i}, q\right)=d_{\mathbb{R}^{2}}\left(\overline{p_{i}}, \bar{q}\right)$. Then we say that $\Delta\left(p_{1}, p_{2}, p_{3}\right)$ satisfies the CAT(0) inequality, if $d_{Y}\left(q_{1}, q_{2}\right) \leq d_{\mathbb{R}^{2}}\left(\overline{q_{1}}, \overline{q_{2}}\right)$ for any pair of points $q_{1}, q_{2}$ on the sides of $\Delta\left(p_{1}, p_{2}, p_{3}\right)$ and their comparison points $\overline{q_{1}}, \overline{q_{2}}$. If every geodesic triangle in $Y$ satisfies the $\mathrm{CAT}(0)$ inequality, then $Y$ is called a $\mathrm{CAT}(0)$ space.

If $Y$ is a $\mathrm{CAT}(0)$ space, it is easy to verify that $Y$ has two important properties: the uniqueness of a geodesic connecting given two points and the contractibility. A complete, simply connected Riemannian manifold of nonpositive sectional curvature is a typical example of CAT(0) space. Also trees and Hilbert spaces are CAT(0) spaces. For an detailed exposition on CAT(0) space, we refer the reader to [3].

Now we recall some definitions which will be necessary later.

Definition 3.1 Let $Y$ be a CAT(0) space.

(1) Let $c$ and $c^{\prime}$ be two nontrivial geodesics in $Y$ starting from $p \in Y$. The angle $\angle_{p}\left(c, c^{\prime}\right)$ between $c$ and $c^{\prime}$ is defined by

$$
\angle_{p}\left(c, c^{\prime}\right)=\lim _{t, t^{\prime} \rightarrow 0} \angle_{\bar{p}}\left(\overline{c(t)}, \overline{c^{\prime}\left(t^{\prime}\right)}\right)
$$

where $\angle_{\bar{p}}\left(\overline{c(t)}, \overline{c^{\prime}\left(t^{\prime}\right)}\right)$ denotes the angle between the sides $\bar{p} \overline{c(t)}$ and $\bar{p} \overline{c^{\prime}\left(t^{\prime}\right)}$ of the comparison triangle $\Delta\left(\bar{p}, \overline{c(t)}, \overline{c^{\prime}\left(t^{\prime}\right)}\right) \subset \mathbb{R}^{2}$.

(2) Let $p \in Y$. For a pair of nontrivial geodesics $c, c^{\prime}$ starting from $p$, we define a relation $\sim$ by $c \sim c^{\prime}$ if and only if $L_{p}\left(c, c^{\prime}\right)=0$. Then $\sim$ becomes an equivalence relation on the set of all nontrivial geodesics starting from $p$ denoted by $\widetilde{\left(S_{p} Y\right)^{\circ}}$. Then the angle $\angle_{p}$ induces a distance on the quotient $\left(S_{p} Y\right)^{\circ}=\widetilde{\left(S_{p} Y\right)^{\circ}} / \sim$, which we denote by the same symbol $\angle_{p}$. The completion $\left(S_{p} Y, \angle_{p}\right)$ of the metric space $\left(\left(S_{p} Y\right)^{\circ}, \angle_{p}\right)$ is called the space of directions at $p$.

(3) Let $T C_{p} Y$ be the cone over $S_{p} Y$, namely,

$$
T C_{p} Y=\left(S_{p} Y \times \mathbb{R}_{\geq 0}\right) /\left(S_{p} Y \times\{0\}\right) .
$$

Let $W, W^{\prime} \in T C_{p} Y$. We may write $W=(V, t)$ and $W^{\prime}=\left(V^{\prime}, t^{\prime}\right)$, where $V, V^{\prime} \in S_{p} Y$ and $t, t^{\prime} \in \mathbb{R}_{\geq 0}$. Then

$$
d_{T C_{p} Y}\left(W, W^{\prime}\right)=t^{2}+t^{\prime 2}-2 t t^{\prime} \cos \angle_{p}\left(V, V^{\prime}\right)
$$

defines a distance on $T C_{p} Y$. The metric space $\left(T C_{p} Y, d_{T C_{p} Y}\right)$ is known to be a CAT(0) space and is called the tangent cone at $p$. We define an "inner product" on $T C_{p} Y$ by

$$
\left\langle W, W^{\prime}\right\rangle=t t^{\prime} \cos \angle_{p}\left(V, V^{\prime}\right) .
$$


We often denote the length $t$ of $W$ by $|W|$; thus we have $|W|=\sqrt{\langle W, W\rangle}=$ $d_{T C_{p} Y}\left(0_{p}, W\right)$, where $0_{p}$ denotes the origin of $T C_{p} Y$.

(4) Define a map $\pi_{p}: Y \longrightarrow T C_{p} Y$ by $\pi_{p}(q)=\left([c], d_{Y}(p, q)\right)$, where $c$ is the geodesic joining $p$ to $q$ and $[c] \in S_{p} Y$ is the equivalence class of $c$. Then $\pi_{p}$ is distance non-increasing.

We often use the following important fact.

Proposition 3.2 ([15, Lemma 2.5.1]) Let $Y$ be a CAT(0) space. Suppose that $\mu$ is a finitely supported measure on $Y$; that is, $\mu$ is a measure of the form $\mu=$ $\sum_{i=1}^{m} \mu_{i} \operatorname{Dirac}_{p_{i}}$, where $\operatorname{Dirac}_{p}$ is the Dirac measure supported on a point $p \in Y$, and $p_{1}, \ldots, p_{m} \in Y$. Then there exists a unique point $p_{0} \in Y$ which minimizes the function

$$
F(q)=\int_{Y} d(p, q)^{2} d \mu(p)=\sum_{i=1}^{m} \mu_{i} d\left(p_{i}, q\right)^{2}, \quad q \in Y .
$$

We call the point $p_{0}$ the barycenter of $\left\{p_{1}, \ldots, p_{m}\right\}$ with respect to a measure $\mu$, or simply barycenter of $\mu$.

Let $Y$ be a $\operatorname{CAT}(0)$ space, and denote by $\operatorname{Isom}(Y)$ the group of isometries of $Y$. Let $\Gamma$ be a finitely generated group, and $\rho: \Gamma \longrightarrow \operatorname{Isom}(Y)$ a homomorphism. We call a map $f: \Gamma \longrightarrow Y \rho$-equivariant if $f$ satisfies $f\left(\gamma \gamma^{\prime}\right)=\rho(\gamma) f\left(\gamma^{\prime}\right)$ for all $\gamma, \gamma^{\prime} \in \Gamma$. Suppose that a $\Gamma$-invariant, symmetric random walk with finite-support property on $\Gamma$ is given. In other word, we are given $\mu: \Gamma \times \Gamma \longrightarrow[0,1]$ with the following properties:

- (finite-support property) For any $\gamma \in \Gamma, \mu\left(\gamma, \gamma^{\prime}\right)=0$ for all but finitely many $\gamma^{\prime} \in \Gamma$,

- For any $\gamma \in \Gamma, \sum_{\gamma^{\prime} \in \Gamma} \mu\left(\gamma, \gamma^{\prime}\right)=1$,

- $\left(\Gamma\right.$-invariance) $\mu\left(\gamma \gamma^{\prime}, \gamma \gamma^{\prime \prime}\right)=\mu\left(\gamma^{\prime}, \gamma^{\prime \prime}\right)$ for any $\gamma, \gamma^{\prime}$, and $\gamma^{\prime \prime} \in \Gamma$,

- (symmetry) $\mu\left(\gamma, \gamma^{\prime}\right)=\mu\left(\gamma^{\prime}, \gamma\right)$ for any $\gamma, \gamma^{\prime} \in \Gamma$.

Then we define the energy $E_{\mu}(f)$ of a $\rho$-equivariant map $f$ by

$$
E_{\mu}(f)=\frac{1}{2} \sum_{\gamma^{\prime} \in \Gamma} \mu\left(\gamma, \gamma^{\prime}\right) d_{Y}\left(f(\gamma), f\left(\gamma^{\prime}\right)\right)^{2} .
$$

Note that, due to the $\Gamma$-invariance of $\mu(\cdot, \cdot)$ and the $\rho$-equivariance of $f$, this value does not depend on the choice of $\gamma$. A $\rho$-equivariant map $f$ is said to be harmonic if $f$ minimizes $E_{\mu}$ among all $\rho$-equivariant maps. Note that the image of a $\rho$-equivariant 
map $f: \Gamma \longrightarrow Y$ is the $\rho(\Gamma)$-orbit of the point $f(e)$, and $f$ is determined by the choice of $f(e)$. Therefore, the set of all $\rho$-equivariant maps from $\Gamma$ to $Y$ can be identified with $Y$. Then the energy functional $E_{\mu}$ becomes a convex continuous function on $Y$, since $Y$ is a $\operatorname{CAT}(0)$ space. Let $-\operatorname{grad} E_{\mu}(f)$ be the negative gradient of the energy functional $E_{\mu}$ at $f$. When $Y$ is a Riemannian manifold this should be understood as the negative of the ordinary gradient. In general, one can give a reasonable definition of $-\operatorname{grad} E_{\mu}(f)$ as an element of the tangent cone of $Y$, identified with the space of $\rho$-equivariant maps, at a point $f$. In fact, $-\operatorname{grad} E_{\mu}(f)$ should be $2(-\Delta f(e))$, where $-\Delta f(e)$ is the barycenter of $\left\{F_{f(e)}(\gamma)=\pi_{f(e)} \circ f(\gamma) \mid \mu(e, \gamma) \neq 0\right\}$ with respect to a measure $\sum_{\gamma} \mu(e, \gamma) \operatorname{Dirac}_{F_{f(e)}(\gamma)}$. The following proposition gives a sufficient condition for the existence of a fixed point of $\rho(\Gamma)$ in terms of the energy functional.

Proposition $3.3([9, \S 1]$, see also $[8, \S 3])$ Let $\Gamma$ be a finitely generated group equipped with a $\Gamma$-invariant, finitely supported, symmetric random walk. Let $Y$ be a CAT(0) space and $\rho: \Gamma \longrightarrow \operatorname{Isom}(Y)$ a homomorphism. Suppose there is a positive constant $C$ such that $|-\Delta f(e)|^{2} \geq C E_{\mu}(f)$ holds for every $\rho$-equivariant map $f$. Then there is a $\rho$-equivariant map $f_{0}$ such that $E_{\mu}\left(f_{0}\right)=0$.

Recall that $\mu$ is called irreducible if $\mu$ satisfies the following: for any $\gamma, \gamma^{\prime} \in \Gamma$, there exists $\gamma_{0}, \gamma_{1}, \ldots, \gamma_{n} \in \Gamma$ such that $\gamma=\gamma_{0}, \gamma^{\prime}=\gamma_{n}$, and $\mu\left(\gamma_{i}, \gamma_{i+1}\right) \neq 0$, $i=0, \ldots, n-1$. If $E_{\mu}\left(f_{0}\right)=0$, then $d\left(f(\gamma), f\left(\gamma^{\prime}\right)\right)=0$ for any pair $\gamma, \gamma^{\prime}$ whenever there exists a sequence $\gamma_{0}, \gamma_{1}, \ldots, \gamma_{n} \in \Gamma$ as above. Therefore, if $\mu$ is irreducible, then $f_{0}$ with $E_{\mu}\left(f_{0}\right)=0$ must be a constant map, and the image of $f_{0}$ is a fixed-point of the action of $\rho(\Gamma)$.

Let $\mu$ be the transition probability of the standard random walk on $\Gamma$ with generating set $S=\left\{s_{1}, \ldots, s_{m}, s_{1}^{-1}, \ldots, s_{m}^{-1}\right\}$, namely,

$$
\mu\left(\gamma, \gamma^{\prime}\right)=\frac{\#\left\{s \in S \mid \gamma^{\prime}=\gamma s\right\}}{2 m} .
$$

We note that $s \in S$ may become the identity element in $\Gamma$, and if this is the case, then $\mu(\gamma, \gamma) \neq 0$ for any $\gamma \in \Gamma$. It is clear that so defined $\mu$ satisfies the four properties listed above and the irreducibility.

Denote by $\mu^{n}$ the $n$th convolution of $\mu$ :

$$
\mu^{n}\left(\gamma, \gamma^{\prime}\right)=\sum_{\gamma_{1} \in \Gamma} \cdots \sum_{\gamma_{n-1} \in \Gamma} \mu\left(\gamma, \gamma_{1}\right) \ldots \mu\left(\gamma_{n-1}, \gamma^{\prime}\right)
$$

We define $n$-step energy $E_{\mu^{n}}(f)$ of a $\rho$-equivariant map $f$ by

$$
E_{\mu^{n}}(f)=\frac{1}{2} \sum_{\gamma^{\prime} \in \Gamma} \mu^{n}\left(\gamma, \gamma^{\prime}\right) d_{Y}\left(f(\gamma), f\left(\gamma^{\prime}\right)\right)^{2} .
$$


Since $\mu^{n}$ is $\Gamma$-invariant as $\mu$, again this does not depend on the choice of $\gamma$.

In [8], Gromov gave an interesting criterion for an action of $\Gamma$ on $Y$ to have a fixed point in terms of the ratio of $E_{\mu}(f)$ and $E_{\mu^{n}}(f)$ (see also [10] and [21]). We need a variant of this criterion, stated as Theorem 3.6 below, in order to prove our main theorems.

A slight modification of the proof of [10, Lemma 2.7(2)] gives the following lemma, which we state in a way convenient for our use.

Lemma 3.4 Let $\Gamma$ be a finitely generated group equipped with the standard random walk, and $Y$ a CAT(0) space. Let $n, k, l$ be natural numbers satisfying $n \geq k l$. Then, for any natural number $i \leq 2 k$, we have

$$
E_{\mu^{2 n-i l}}(f) \leq(2 k-i+1)^{2}\left(E_{\mu^{l}}(f)+E_{\mu^{2 n-2 k l}}(f)\right)
$$

and

$$
\left|-\Delta_{2 n-i l} f(e)\right| \leq(2 k-i+1) \sqrt{2\left(E_{\mu^{l}}(f)+E_{\mu^{2 n-2 k l}}(f)\right)},
$$

where $-\Delta_{j} f(e)$ is the barycenter of $\left\{F_{f(e)}(\gamma)=\pi_{f(e)} \circ f(\gamma) \mid \mu^{j}(e, \gamma) \neq 0\right\}$ with respect to a measure $\sum_{\gamma} \mu^{j}(e, \gamma) \operatorname{Dirac}_{F_{f(e)}(\gamma)}$.

Proof Noting $\mu^{2 n-i l}=\left(\mu^{l}\right)^{2 k-i} * \mu^{2 n-2 k l}$, we get

$$
\begin{aligned}
& E_{\mu^{2 n-i l}}(f) \\
= & \frac{1}{2} \sum_{\gamma_{1}, \ldots, \gamma_{2 k-i+1}} \mu^{l}\left(e, \gamma_{1}\right) \cdots \mu^{l}\left(\gamma_{2 k-i-1}, \gamma_{2 k-i}\right) \mu^{2 n-2 k l}\left(\gamma_{2 k-i}, \gamma_{2 k-i+1}\right) \\
& \times d_{Y}\left(f(e), f\left(\gamma_{2 k-i+1}\right)\right)^{2} \\
\leq & \frac{1}{2} \sum_{\gamma_{1}, \ldots, \gamma_{2 k-i+1}} \mu^{l}\left(e, \gamma_{1}\right) \cdots \mu^{l}\left(\gamma_{2 k-i-1}, \gamma_{2 k-i}\right) \mu^{2 n-2 k l}\left(\gamma_{2 k-i}, \gamma_{2 k-i+1}\right) \\
& \times(2 k-i+1)\left(d_{Y}\left(f(e), f\left(\gamma_{1}\right)\right)^{2}+\cdots+d_{Y}\left(f\left(\gamma_{2 k-i}\right), f\left(\gamma_{2 k-i+1}\right)\right)^{2}\right) .
\end{aligned}
$$


Note that

$$
\begin{aligned}
& \frac{1}{2} \sum_{\gamma_{1}, \ldots, \gamma_{2 k-i+1}} \mu^{l}\left(e, \gamma_{1}\right) \ldots \mu^{2 n-2 k l}\left(\gamma_{2 k-i}, \gamma_{2 k-i+1}\right) d_{Y}\left(f\left(\gamma_{2 k-i}\right), f\left(\gamma_{2 k-i+1}\right)\right)^{2} \\
= & \frac{1}{2} \sum_{\gamma_{1}, \ldots, \gamma_{2 k-1}} \mu^{l}\left(e, \gamma_{1}\right) \ldots \mu^{l}\left(\gamma_{2 k-i-1}, \gamma_{2 k-i}\right) \\
& \times \sum_{\gamma_{2 k-i+1}} \mu^{2 n-2 k l}\left(\gamma_{2 k-i}, \gamma_{2 k-i+1}\right) d_{Y}\left(f\left(\gamma_{2 k-i}\right), f\left(\gamma_{2 k-i+1}\right)\right)^{2} \\
= & \sum_{\gamma_{1}, \ldots, \gamma_{2 k-i}} \mu^{l}\left(e, \gamma_{1}\right) \ldots \mu^{l}\left(\gamma_{2 k-i-1}, \gamma_{2 k-i}\right) \times E_{\mu^{2 n-2 k l}}(f) \\
= & E_{\mu^{2 n-2 k l}}(f),
\end{aligned}
$$

and, for $j<2 k-i$,

$$
\begin{aligned}
& \frac{1}{2} \sum_{\gamma_{1}, \ldots, \gamma_{2 k-i+1}} \mu^{l}\left(e, \gamma_{1}\right) \ldots \mu^{2 n-k l}\left(\gamma_{2 k-i}, \gamma_{2 k-i+1}\right) d_{Y}\left(f\left(\gamma_{j}\right), f\left(\gamma_{j+1}\right)\right)^{2} \\
= & \frac{1}{2} \sum_{\gamma_{1}, \ldots, \gamma_{2 k-i}} \mu^{l}\left(e, \gamma_{1}\right) \ldots \mu^{l}\left(\gamma_{j}, \gamma_{j+1}\right) \ldots \mu^{l}\left(\gamma_{2 k-i-1}, \gamma_{2 k-i}\right) \\
& \times \sum_{\gamma_{2 k-i+1}} \mu^{2 n-2 k l}\left(\gamma_{2 k-i}, \gamma_{2 k-i+1}\right) d_{Y}\left(f(e), f\left(\gamma_{j}^{-1} \gamma_{j+1}\right)\right)^{2} \\
= & \frac{1}{2} \sum_{\gamma_{1}, \ldots, \gamma_{2 k-i-1}} \mu^{l}\left(e, \gamma_{1}\right) \ldots \mu^{l}\left(\gamma_{j}, \gamma_{j+1}\right) \ldots \mu^{l}\left(\gamma_{2 k-i-2}, \gamma_{2 k-i-1}\right) \\
& \times \sum_{\gamma_{2 k-i}} \mu^{l}\left(\gamma_{2 k-i-1}, \gamma_{2 k-i}\right) d_{Y}\left(f\left(\gamma_{j}\right), f\left(\gamma_{j+1}\right)\right)^{2} \\
= & \frac{1}{2} \sum_{\gamma_{1}, \ldots, \gamma_{j}} \mu^{l}\left(e, \gamma_{1}\right) \ldots \mu^{l}\left(\gamma_{j-1}, \gamma_{j}\right) \times \sum_{\gamma_{j+1}} \mu^{l}\left(\gamma_{j}, \gamma_{j+1}\right) d_{Y}\left(f\left(\gamma_{j}\right), f\left(\gamma_{j+1}\right)\right)^{2} \\
= & \sum_{\gamma_{1}} \ldots \sum_{\gamma_{j}} \mu^{l}\left(e, \gamma_{1}\right) \ldots \mu^{l}\left(\gamma_{j-1}, \gamma_{j}\right) E_{\mu^{l}}(f) \\
= & E_{\mu^{l}}(f)
\end{aligned}
$$

holds. Together with (3-3), this implies (3-2):

$$
\begin{aligned}
E_{\mu^{2 n-i l}}(f) & \leq(2 k-i+1)\left((2 k-i) E_{\mu^{l}}(f)+E_{\mu^{2 n-2 k l}}(f)\right) \\
& \leq(2 k-i+1)^{2}\left(E_{\mu^{l}}(f)+E_{\mu^{2 n-2 k l}}(f)\right) .
\end{aligned}
$$

Since $\left|-\Delta_{2 n-i l} f(e)\right| \leq \sqrt{2 E_{\mu^{2 n-i l}}(f)}$ follows from [10, Lemma 2.7(1)], combining with (3-2) implies the other inequality.

The following is a variant of [10, Proposition 2.8]. 
Proposition 3.5 Let $\Gamma$ be a finitely generated group equipped with the standard random walk, and $Y$ a $\mathrm{CAT}(0)$ space. If natural numbers $n, k$ and $l$ satisfy $n \geq k l$, we have

$$
\begin{aligned}
E_{\mu^{2 n}}(f) \geq & E_{\mu^{2 n-2 k l}}(f)+2 k E_{\mu^{l}}(f) \\
& -\sum_{i=1}^{2 k}(2 k-i+1) \sqrt{2\left(E_{\mu^{l}}(f)+E_{\mu^{2 n-2 k l}}(f)\right)}\left|-\Delta_{l} f(e)\right| .
\end{aligned}
$$

Proof By using [10, Proposition 2.5] $2 k$ times, we obtain

$$
\begin{aligned}
E_{\mu^{2 n}}(f) \geq & E_{\mu^{2 n-l}}(f)+E_{\mu^{l}}(f)-\left\langle-\Delta_{2 n-l} f(e),-\Delta_{l} f(e)\right\rangle \\
\geq & \left(E_{\mu^{2 n-2 l}}(f)+E_{\mu^{l}}(f)-\left\langle-\Delta_{2 n-2 l} f(e),-\Delta_{l} f(e)\right\rangle\right) \\
& +E_{\mu^{l}}(f)-\left\langle-\Delta_{2 n-l} f(e),-\Delta_{l} f(e)\right\rangle \\
& \vdots \\
\geq & E_{\mu^{2 n-2 k l}}(f)+2 k E_{\mu^{l}}(f)-\sum_{i=1}^{2 k}\left\langle-\Delta_{2 n-i l} f(e),-\Delta_{l} f(e)\right\rangle \\
\geq & E_{\mu^{2 n-2 k l}}(f)+2 k E_{\mu^{l}}(f)-\sum_{i=1}^{2 k}\left|-\Delta_{2 n-i l} f(e)\right|\left|-\Delta_{l} f(e)\right| .
\end{aligned}
$$

Now Lemma 3.4 implies the desired inequality.

Theorem 3.6 Let $\Gamma$ be a finitely generated group equipped with the standard random walk, and $Y$ a CAT(0) space. Suppose that natural numbers $n, k$ and $l$ satisfy $n \geq k l$, and that there exists $\varepsilon>0$ such that, for any $\rho$-equivariant map $f$, the following inequality holds:

$$
E_{\mu^{2 n}}(f) \leq(2 k-\varepsilon) E_{\mu^{l}}(f) .
$$

Then there is a $\rho$-equivariant map $f_{0}$ with $E_{\mu^{l}}\left(f_{0}\right)=0$. Moreover, $\rho(\Gamma)$ admits a fixed point.

Proof By (3-4) and the assumption, we get

$$
\begin{aligned}
(2 k-\varepsilon) E_{\mu^{l}}(f) \geq & E_{\mu^{2 n-2 k l}}(f)+2 k E_{\mu^{l}}(f) \\
& -\sum_{i=1}^{2 k}(2 k-i+1) \sqrt{2\left(E_{\mu^{l}}(f)+E_{\mu^{2 n-2 k l}}(f)\right)}\left|-\Delta_{l} f(e)\right|,
\end{aligned}
$$


and hence

$$
\begin{aligned}
& c(k)\left|-\Delta_{l} f(e)\right| \sqrt{E_{\mu^{l}}(f)+E_{\mu^{2 n-2 k l}}(f)} \\
\geq & \varepsilon E_{\mu^{l}}(f)+E_{\mu^{2 n-2 k l}}(f) \\
\geq & \varepsilon\left(E_{\mu^{l}}(f)+E_{\mu^{2 n-2 k l}}(f)\right),
\end{aligned}
$$

since we may assume $\varepsilon \leq 1$. Hence

$$
\begin{aligned}
\left|-\Delta_{l} f(e)\right| & \geq \frac{\varepsilon}{c(k)} \sqrt{E_{\mu^{l}}(f)+E_{\mu^{2 n-2 k l}}(f)} \\
& \geq \frac{\varepsilon}{c(k)} \sqrt{E_{\mu^{l}}(f) .}
\end{aligned}
$$

By Proposition 3.3, this implies the existence of $f_{0}$ with $E_{\mu^{l}}\left(f_{0}\right)=0$. If $l$ is odd, then $\mu^{l}(e, s) \neq 0$ for any $s \in S$. Thus $f_{0}(s)=f_{0}(e)$ for any $s \in S$. Since $f_{0}$ is $\rho$-equivariant, this implies that $f_{0}(\gamma)=f_{0}(e)$ for any $\gamma \in \Gamma$, which means that $f_{0}$ is a constant map and $f_{0}(e)$ is a fixed point. Suppose $l$ is even. Let $\Gamma^{\prime}$ be a subgroup of $\Gamma$ generated by $S^{\prime}=\left\{\gamma \in \Gamma \mid \mu^{2}(e, \gamma) \neq 0\right\}$. Then $\mu^{l}(e, \gamma) \neq 0$ for any $\gamma \in S^{\prime}$, and $f_{0}(e)$ is fixed by $\rho\left(\Gamma^{\prime}\right)$. On the other hand,

$$
\varphi(\gamma)= \begin{cases}0 & \text { if } \gamma \text { can be expressed by word with even length, } \\ 1 & \text { otherwise }\end{cases}
$$

defines a homomorphism $\varphi: \Gamma \longrightarrow \mathbb{Z} / 2 \mathbb{Z}$, and the kernel is exactly $\Gamma^{\prime}$. Therefore, $\Gamma^{\prime}$ is a subgroup of index at most 2. Since $f_{0}\left(\Gamma^{\prime}\right)$ is a point and the image $f_{0}(\Gamma)$ is the $\rho(\Gamma)$-orbit of $f_{0}\left(\Gamma^{\prime}\right), f_{0}(\Gamma)$ consists of at most two points. In other words, we have an orbit consisting of at most two points. If the orbit consists of a single point, then the point is a fixed point for the action of $\rho(\Gamma)$. Suppose the orbit consists of two points. In this case, the action $\rho(\Gamma)$ is simply a permutation of these two points; if $\gamma \in \Gamma^{\prime}$, then $\rho(\gamma)$ must fix both points, while if $\gamma \notin \Gamma^{\prime}$, then $\rho(\gamma)$ exchanges these two points. Since the action of $\rho(\Gamma)$ is by isometry, their unique midpoint must be fixed by $\rho(\Gamma)$. (The uniqueness of the midpoint follows from the uniqueness of a geodesic joining given two points, which is true because $Y$ is a $\operatorname{CAT}(0)$ space.) This completes the proof.

According to Theorem 3.6, in order to prove the fixed-point property of $\Gamma$ for $Y$, we need to give a bound on the ratio $E_{\mu^{2 n}}(f) / E_{\mu^{l}}(f)$, which is less than $2 k$ and independent of $\rho$ and $f$. The ratio $E_{\mu^{2 n}}(f) / E_{\mu^{l}}(f)$ has an interesting connection with the Rayleigh quotient of a map from a graph into $Y$ as we explain below.

Let $\Gamma$ be the free group generated by $S: \Gamma=\left\langle s_{1}\right\rangle * \cdots *\left\langle s_{m}\right\rangle$. Let $\Gamma_{P}$ be the group given by a presentation $P=(S, R) \in P(m, \eta, d)$. Let $G_{P}=\left(V, E_{P}\right)$ be the graph 
associated to $P$ defined in $\S 2$. Let $Y$ be a CAT(0) space with a metric $d=d_{Y}$, and $f: V \longrightarrow Y$ a map into $Y$. Then the Rayleigh quotient $R Q^{G}(f)$ of $f$, introduced by Gromov, is defined as

$$
R Q^{G}(f)=\frac{\|d f\|_{L^{2}}^{2}}{F(f)}
$$

where

$$
\left\{\begin{array}{l}
\|d f\|_{L^{2}}^{2}=\frac{1}{2} \sum_{v \in V} \sum_{v^{\prime} \in V} \nu\left(v, v^{\prime}\right) d_{Y}\left(f(v), f\left(v^{\prime}\right)\right)^{2} \\
F(f)=\frac{1}{2} \sum_{v, v^{\prime} \in V} \nu(v) \nu\left(v^{\prime}\right) d_{Y}\left(f(v), f\left(v^{\prime}\right)\right)^{2} .
\end{array}\right.
$$

When $Y=\mathbb{R}$ this is nothing but the Rayleigh quotient introduced in $\S 2$. For a general $\mathrm{CAT}(0)$ space $Y$, using the triangle inequality, one sees that

$$
\begin{aligned}
F(f) & \leq \frac{1}{2} \sum_{v, v^{\prime} \in V} \nu(v) \nu\left(v^{\prime}\right)\left(2 d_{Y}(\operatorname{bar}(f), f(v))^{2}+2 d_{Y}\left(\operatorname{bar}(f), f\left(v^{\prime}\right)\right)^{2}\right) \\
& =2 \sum_{v \in V} d_{Y}(\operatorname{bar}(f), f(v)),
\end{aligned}
$$

where $\operatorname{bar}(f)$ is the barycenter of $f(V)$. Let $\delta(Y)$ be the invariant introduced in [11, Definition 6.1], and $\lambda_{1}(G, Y)$ be Wang's invariant (see [22] or [11, Definition 4.2]). Then by [11, Proposition 6.3], for any nonconstant map $f: V \longrightarrow Y$, we obtain

$$
\begin{aligned}
R Q^{G}(f) & \geq \frac{1}{2} \frac{\|d f\|_{L^{2}}^{2}}{\sum_{v \in V} d_{Y}(\operatorname{bar}(f), f(v))} \\
& \geq \frac{1}{2} \lambda_{1}(G, Y) \geq \frac{1}{2}(1-\delta(Y)) \lambda_{1}(G) .
\end{aligned}
$$

Let $\rho: \Gamma_{P} \longrightarrow \operatorname{Isom}(Y)$ be a homomorphism, and take any $\rho$-equivariant map $f: \Gamma_{P} \longrightarrow Y$. Then we can consider $\tilde{\rho}$-equivariant map $\tilde{f}=f \circ \pi_{P}: \Gamma \longrightarrow Y$, where $\pi_{P}$ denotes the projection from $\Gamma$ onto $\Gamma_{P}$ and $\tilde{\rho}=\rho \circ \pi_{P}: \Gamma \longrightarrow \operatorname{Isom}(Y)$. Regarding $v \in V$ as an element $\pi_{P}(\bar{v}) \in \Gamma_{P}$ in a natural way, we get a map $\bar{f}: V \longrightarrow Y$ induced from $f$, where $\bar{v}$ denotes the element in $\Gamma$ determined by a word $v$.

Regarding a $\rho$-equivariant map as a map defined on $V$ as above, we will be able to show that the Rayleigh quotient approximates the ratio of $l$-step and $2 n$-step energies with high probability.

Proposition 3.7 Fix a natural number $k$, and let $k /(2 k+1)<d<1 / 2$. Set $l$ and $n$ as described in (2-1). Then, for any $\varepsilon>0$, we have

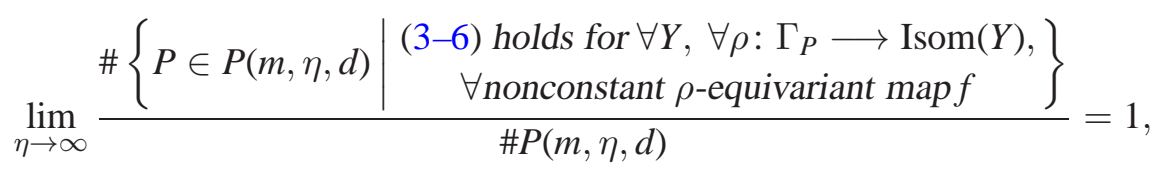


where

$$
(1-\varepsilon) R Q^{G}(\bar{f}) \leq \frac{E_{\mu^{l}}(\tilde{f})}{E_{\mu^{2 n}}(\tilde{f})} \leq(1+\varepsilon) R Q^{G}(\bar{f})
$$

and $Y$ denotes a $\mathrm{CAT}(0)$ space.

The proof will be given in $\S 6.2$.

Remark 1 We are assuming $d<1 / 2$ in order to regard $P(m, \eta, d)$ as the probability space describing an experiment given by choosing a word $c_{0}(2 m)^{d \eta}$ times randomly from $W_{\eta}$, where $c^{-1} \leq c_{0} \leq c$. However, the assumption $d<1 / 2$ is not essential as we will explain in Remark 2.

\section{Proofs of Theorem 1.1 and 1.2}

We first give the proof of Theorem 1.2, assuming Proposition 2.1 and 3.7 and then point out a necessary change in order to show Theorem 1.1.

Note that $1-\frac{1}{2} \log _{2 m}(8 m-4)<1 / 2$ holds, and that we have $R Q^{G}(f) \geq \lambda_{1}\left(G_{P}, Y\right) \geq$ $\frac{1}{2}(1-\delta(Y)) \lambda_{1}\left(G_{P}\right) \geq \frac{1}{2}(1-\delta) \lambda_{1}\left(G_{P}\right)$ for any $Y \in \mathcal{Y}_{\delta}$ by (3-5) and our assumption. By Propositions 2.1 and 3.7, we see that if $k /(2 k+1)<d<1-\frac{1}{2} \log _{2 m}(8 m-4)$, with probability tending to 1 as $\eta \rightarrow \infty, \Gamma_{P}$ satisfies

$$
\frac{E_{\mu^{l}}(\tilde{f})}{E_{\mu^{2 n}}(\tilde{f})} \geq \frac{1-\delta}{2}(1-\varepsilon)
$$

for any $Y \in \mathcal{Y}_{\delta}, \rho: \Gamma_{P} \longrightarrow \operatorname{Isom}(Y)$, and $\tilde{\rho}$-equivariant map $\tilde{f}$, where $\tilde{\rho}=\rho \circ$ $\pi_{P}: \Gamma \longrightarrow \operatorname{Isom}(Y)$. Rewriting this, we see that, for any $\varepsilon^{\prime}>0, P \in P(m, \eta, d)$ satisfies the following property with high probability, that is, probability tending to 1 as $\eta \rightarrow \infty$ : For any $Y \in \mathcal{Y}_{\delta}, \rho: \Gamma_{P} \longrightarrow \operatorname{Isom}(Y)$, and for any $\tilde{\rho}$-equivariant map $\tilde{f}: \Gamma \longrightarrow Y$,

$$
E_{\mu^{2 n}}(\tilde{f}) \leq \frac{2}{1-\delta}\left(1+\varepsilon^{\prime}\right) E_{\mu^{l}}(\tilde{f})
$$

holds. Since we have $k>1 /(1-\delta)$, by taking $\varepsilon^{\prime}$ to be sufficiently small, we may assume

$$
\frac{2}{1-\delta}\left(1+\varepsilon^{\prime}\right) \leq\left(2 k-\varepsilon^{\prime \prime}\right)
$$

for some $\varepsilon^{\prime \prime}>0$. Therefore, we obtain, for any $\rho: \Gamma_{P} \longrightarrow \operatorname{Isom}(Y)$ and $\tilde{\rho}$-equivariant $\operatorname{map} \tilde{f}$,

$$
E_{\mu^{2 n}}(\tilde{f}) \leq\left(2 k-\varepsilon^{\prime \prime}\right) E_{\mu^{l}}(\tilde{f})
$$


and hence $\rho\left(\Gamma_{P}\right)=\tilde{\rho}(\Gamma)$ admits a fixed point by Theorem 3.6. This implies fixed-point property of $\Gamma_{P}$ with high probability. This completes the proof of Theorem 1.2.

If $Y=\mathcal{H}$, then we have $R Q^{G}(f) \geq \lambda_{1}\left(G_{P}\right)$. Therefore we have

$$
\frac{E_{\mu^{l}}(\tilde{f})}{E_{\mu^{2 n}}(\tilde{f})} \geq 1-\varepsilon
$$

instead of (4-1), and hence

$$
E_{\mu^{2 n}}(\tilde{f}) \leq\left(1+\varepsilon^{\prime}\right) E_{\mu^{l}}(\tilde{f}) .
$$

Now we need $1+\varepsilon^{\prime} \leq\left(2 k-\varepsilon^{\prime \prime}\right)$ in order to apply Theorem 3.6, and it suffices to take $k=1$. Therefore taking $d>1 /(2+1)=1 / 3$ is sufficient, and this completes the proof of Theorem 1.1.

\section{Preliminaries on probability theory}

In this section, we collect some facts from probability theory, which will be used in the proof of Propositions 2.1 and 3.7.

\subsection{Notation and some inequalities}

Let $X$ be a random variable defined on a probability space $(\Omega, P)$. We denote the probability of $X$ taking its value in a set $A$ by $\mathbb{P}(X \in A)$ :

$$
\mathbb{P}(X \in A)=\int_{\{\omega: X(\omega) \in A\}} d P(\omega)
$$

Suppose $X$ is nonnegative and let $\mu$ be the mean (expected value $\mathbb{E}(X)$ ) of $X$. Then, for $t>0$,

$$
\mu=\int X d P \geq \int_{X \geq t \mu} X d P \geq \int_{X \geq t \mu} t \mu d P=\mathbb{P}(X \geq t \mu) t \mu .
$$

Dividing the both ends by $t \mu$ yields Markov's inequality:

$$
\mathbb{P}(X \geq t \mu) \leq \frac{1}{t} .
$$

Setting $\varepsilon=t \mu$ gives

$$
\mathbb{P}(X \geq \varepsilon) \leq \frac{\mu}{\varepsilon} .
$$


If $X$ is a real valued random variable with mean $\mu$ and variance $\sigma^{2}$. Then, for any $d>0$,

$$
\sigma^{2}=\mathbb{E}\left((X-\mu)^{2}\right) \geq \int_{|X-\mu| \geq d}(X-\mu)^{2} d P \geq \mathbb{P}(|X-\mu| \geq d) d^{2} .
$$

Dividing the both ends by $d^{2}$, we obtain Chebyshev's inequality:

$$
\mathbb{P}(|X-\mu| \geq d) \leq \frac{\sigma^{2}}{d^{2}} .
$$

By taking $d=\varepsilon \mu$, we get

$$
\mathbb{P}(|X-\mu| \geq \varepsilon \mu) \leq \frac{\sigma^{2}}{\varepsilon^{2} \mu^{2}} .
$$

Let $X$ be a binomially distributed random variable with size $n$ and parameter $p$; namely, $X$ satisfies

$$
\mathbb{P}(X=x)={ }_{n} C_{x} p^{x}(1-p)^{n-x}, \quad x=0,1, \ldots, n .
$$

Then we say that $X$ has the binomial distribution $\operatorname{Bi}(n, p)$. Such an $X$ has mean $\mu=n p$ and variance $\sigma^{2}=n p(1-p)$. Thus Chebyshev's inequality yields

$$
\mathbb{P}(|X-\mu| \geq \varepsilon \mu) \leq \frac{n p(1-p)}{\varepsilon^{2} n^{2} p^{2}}=\frac{1-p}{\varepsilon^{2} \mu} \leq \frac{1}{\varepsilon^{2} \mu} .
$$

In particular, we see that if $\left\{X_{i}\right\}$ is a sequence of binomially distributed random variable with mean $\mu_{i} \rightarrow \infty$, then $\mathbb{P}\left(\left|X_{i}-\mu_{i}\right| \geq \varepsilon \mu_{i}\right) \rightarrow 0$. Actually, there are finer and more useful bounds called Chernoff bounds:

$$
\begin{aligned}
& \mathbb{P}(X \geq \mu+t) \leq \exp \left(-\frac{t^{2}}{2(\mu+t / 3)}\right) \\
& \mathbb{P}(X \leq \mu-t) \leq \exp \left(-\frac{t^{2}}{2 \mu}\right) .
\end{aligned}
$$

See, for example, $[12, \S 2.1]$ for proofs. In particular, taking $t=\varepsilon \mu$ with $0<\varepsilon<1$, we obtain

$$
\mathbb{P}(|X-\mu|>\varepsilon \mu) \leq 2 \exp \left(-\frac{\varepsilon^{2} \mu}{3}\right)
$$

\subsection{Moment generating function for binomially distributed random vari- ables}

Let $X$ be a random variable on a probability space. Then

$$
\mathbb{E}(\exp (t X))=\mathbb{E}\left(\sum_{k=0}^{\infty} \frac{1}{k !} t^{k} X^{k}\right)=\sum_{k=0}^{\infty} \frac{1}{k !} t^{k} \mathbb{E}\left(X^{k}\right)
$$


is called the moment generating function of $X$. We can compute $\alpha$ th moment $\mathbb{E}\left(X^{\alpha}\right)$ of $X$ by

$$
\mathbb{E}\left(X^{\alpha}\right)=\left.\frac{d^{\alpha}}{d t^{\alpha}} \mathbb{E}(\exp (t X))\right|_{t=0} .
$$

Let $X$ be a random variable with distribution $\operatorname{Bi}(n, p)$. Then the mean of $X$ is $n p$, and by computing the average, we get

$$
\begin{aligned}
\mathbb{E}(\exp t(X-n p)) & =\sum_{x=0}^{n} \exp t(x-n p){ }_{n} C_{x} p^{x} q^{n-x} \\
& =\sum_{x=0}^{n}\left({ }_{n} C_{x}\left(e^{t} p\right)^{x} q^{n-x}\right) e^{-t n p} \\
& =\left(e^{t} p+q\right)^{n} e^{-t n p},
\end{aligned}
$$

where $q=1-p$. A computation shows the following.

Lemma 5.1 Let $X$ be a random variable with distribution $\operatorname{Bi}(n, p)$, and set $M(t)=$ $\mathbb{E}(\exp t(X-n p))$. Then, $\alpha$ th derivative $M^{(\alpha)}(t)$ of $M(t)$ can be computed as

$$
M^{(\alpha)}(t)=\sum_{\beta=0}^{\alpha}{ }_{\alpha} C_{\beta}\left[\left(e^{t} p+q\right)^{n}\right]^{(\beta)}\left[e^{-t n p}\right]^{(\alpha-\beta)},
$$

where $[\cdot]^{(a)}$ denotes the ath derivative of $[\cdot]$ and they turned out to be

$$
\begin{aligned}
& {\left[\left(e^{t} p+q\right)^{n}\right]^{(\beta)}=\sum_{j=1}^{\beta} c(\beta, j) \frac{n !}{(n-j) !}\left(e^{t} p+q\right)^{n-j} e^{j t} p^{j} \quad(\beta>0),} \\
& {\left[e^{-t n p}\right]^{(\alpha-\beta)}=(-n p)^{\alpha-\beta} e^{-t n p} .}
\end{aligned}
$$

Here $c(k, j)$ is determined by $c(j, j)=1, c(j, 0)=0$, and $c(k+1, j)=c(k, j-1)+$ $j c(k, j)$.

We will use the following estimate in the proof of Proposition 2.1.

Lemma 5.2 For a random variable $X$ with distribution $\operatorname{Bi}(n, p)$ and with mean $\mu=$ $n p<1$, we have

$$
\left|\mathbb{E}\left((X-n p)^{\alpha}\right)\right| \leq c(\alpha) n p
$$

Proof Since $\mathbb{E}\left((X-n p)^{\alpha}\right)=M^{(\alpha)}(0)$ as we have seen above, we need to estimate $M^{(\alpha)}(0)$. By Lemma 5.1, and $n p<1$, if $\beta>0$

$$
\left|\left[\left(e^{t} p+q\right)^{n}\right]^{(\beta)}\right|_{t=0} \mid \leq \sum_{j=1}^{\beta} c(\beta, j) \frac{n !}{(n-j) !} p^{j} \leq \sum_{j=1}^{\beta} c(\beta, j) n^{j} p^{j} \leq \sum_{j=1}^{\beta} c(\beta, j) n p,
$$


and if $\alpha>\beta$

$$
\left|\left[e^{-t n p}\right]^{(\alpha-\beta)}\right|_{t=0} \mid \leq(n p)^{\alpha-\beta} \leq n p .
$$

Thus we obtain the desired estimate.

\subsection{Remarks on our model}

In order to prove Propositions 2.1 and 3.7, it is convenient to fix the cardinality of $R$, the set of relations, and consider

$$
P\left(m, \eta, d, c_{0}\right)=\left\{P=(S, R) \in P(m, \eta, d) \mid \# R=c_{0}(2 m)^{d \eta}\right\},
$$

where $c^{-1} \leq c_{0} \leq c$. Then the model $P\left(m, \eta, d, c_{0}\right)$ can be regarded as the set of subsets of $W_{\eta}$ with cardinality $c_{0}(2 m)^{d \eta}$ equipped with the uniform counting measure. Of course, this is not the same as the probability space $P^{\prime}\left(m, \eta, d, c_{0}\right)$ which describes an experiment given by choosing a word $c_{0}(2 m)^{d \eta}$ times randomly from $W_{\eta}$; there may be multiply chosen words and there is a natural action of the permutation group given by changing the order of the choice. If we take $d<1 / 2$, then the probability of an elements in $P^{\prime}\left(m, \eta, d, c_{0}\right)$ to have multiply chosen words tends to 0 as $\eta \rightarrow$ $\infty$ as the following computation shows. Note that, on the subset $P_{0}^{\prime}\left(m, \eta, d, c_{0}\right)$ of $P^{\prime}\left(m, \eta, d, c_{0}\right)$ consisting of elements without multiply chosen words, the action of the permutation group mentioned above is free. Thus, taking the quotient by the action of the permutation group, we can regard $P_{0}^{\prime}\left(m, \eta, d, c_{0}\right)$ as $P\left(m, \eta, d, c_{0}\right)$.

If $X$ is a random variable with distribution $\operatorname{Bi}(n, p)$, then

$$
\mathbb{P}(X \geq 2)=1-\mathbb{P}(X=0 \text { or } 1)=1-\left((1-p)^{n}+n(1-p)^{n-1} p\right) .
$$

Since

$$
\begin{aligned}
& (1-p)^{n}+n(1-p)^{n-1} p \\
= & \sum_{\alpha=0}^{n}{ }_{n} C_{\alpha} 1^{n-\alpha}(-p)^{\alpha}+n p \sum_{\alpha=0}^{n-1}{ }_{n-1} C_{\alpha} 1^{n-\alpha}(-p)^{\alpha} \\
\geq & 1-n p-n^{2} p^{2}-\cdots-n^{n} p^{n} \\
& \quad+n p\left(1-(n-1) p-(n-1)^{2} p^{2}-\cdots-(n-1)^{n-1} p^{n-1}\right) \\
\geq & 1-2(n p)^{2}-\cdots-2(n p)^{n}
\end{aligned}
$$

we obtain

$$
\mathbb{P}(X \geq 2) \leq 2 \sum_{\alpha=2}^{n}(n p)^{\alpha}
$$


Now take any $w \in W_{\eta}$, and denote by $X_{w}(P)$ the number of times that $w$ is chosen in an element $P \in P^{\prime}\left(m, \eta, d, c_{0}\right)$. Then $X_{w}$ is a random variable on $P^{\prime}\left(m, \eta, d, c_{0}\right)$ with distribution $\operatorname{Bi}\left(c_{0}(2 m)^{d \eta},(2 m)^{-\eta}\right)$. By the computation above, we have

$$
\mathbb{P}\left(X_{w} \geq 2\right) \leq 2 \sum_{\alpha=2}^{c_{0}(2 m)^{d \eta}}\left(c_{0}(2 m)^{d \eta}(2 m)^{-\eta}\right)^{\alpha}
$$

Since $\# W_{\eta}=(2 m)^{\eta}$, we see that

$$
\begin{aligned}
\mathbb{P}\left(X_{w} \geq 2 \text { for some } w \in W_{\eta}\right) & \leq(2 m)^{\eta} \times 2 \sum_{\alpha=2}^{c_{0}(2 m)^{d \eta}} c_{0}^{\alpha}(2 m)^{\alpha d \eta}(2 m)^{-\alpha \eta} \\
& =2 \sum_{\alpha=2}^{c_{0}(2 m)^{d \eta}} c_{0}^{\alpha}(2 m)^{\alpha d \eta}(2 m)^{-(\alpha-1) \eta} \\
& =2 c_{0}^{2}(2 m)^{(2 d-1) \eta} \sum_{\alpha=2}^{c_{0}(2 m)^{d \eta}} c_{0}^{\alpha-2}(2 m)^{(\alpha-2)(d-1) \eta} \\
& \leq 2 c_{0}^{2}(2 m)^{(2 d-1) \eta} \frac{1}{1-c_{0}(2 m)^{-(1-d) \eta}}
\end{aligned}
$$

holds for large $\eta$, since $c_{0}(2 m)^{-(1-d) \eta}<1$ when $\eta$ is large enough. By taking $d<1 / 2$ and suitable constant $c^{\prime}$, we see that

$$
\mathbb{P}\left(X_{w} \geq 2 \text { for some } w \in W_{\eta}\right) \leq c^{\prime}(2 m)^{(2 d-1) \eta} \rightarrow 0 \quad(\eta \rightarrow \infty) .
$$

Note that $c^{\prime}$ can be taken so that depending only on $c$ taken in order to define $P(m, \eta, d)$ but independent of $c_{0}$. In other words, the measure of $P_{0}^{\prime}\left(m, \eta, d, c_{0}\right)$ in the probability space $P^{\prime}\left(m, \eta, d, c_{0}\right)$ uniformlly tends to 1 . Hence, assuming $d<1 / 2$, we may identify $P\left(m, \eta, d, c_{0}\right)$ with $P^{\prime}\left(m, \eta, d, c_{0}\right)$. We will use this identification in the proof of Proposition 3.7.

In order to prove Proposition 2.1, we need another model $P^{\prime \prime}\left(m, \eta, d, c_{0}\right)$ of random groups. In this model $P^{\prime \prime}\left(m, \eta, d, c_{0}\right)$, each word in $W_{\eta}$ is independently chosen to be a relation of $P=(S, R)$ with probability $\left(c_{0}(2 m)^{d \eta}\right) /(2 m)^{\eta}$. Therefore any subset of $W_{\eta}$ can occur as $R$ of $P=(S, R)$ in $P^{\prime \prime}\left(m, \eta, d, c_{0}\right)$, and the probability of the occurrence is given by

$$
\mathbb{P}(P=(S, R))=\left(\frac{c_{0}(2 m)^{d \eta}}{(2 m)^{\eta}}\right)^{\# R}\left(1-\frac{c_{0}(2 m)^{d \eta}}{(2 m)^{\eta}}\right)^{(2 m)^{\eta}-\# R} .
$$

This model corresponds to a model of random graphs often denoted by $G(n, p)$ in the literature, where $n=(2 m)^{\eta}$ and $p=\left(c_{0}(2 m)^{d \eta}\right) /(2 m)^{\eta}$ in our case; while our original model $P\left(m, \eta, d, c_{0}\right)$ corresponds to another model of random graphs often denoted 
by $G(n, M)$, where $n=(2 m)^{\eta}$ and $M=c_{0}(2 m)^{d \eta}$ in our case. In our setting, a well-known relation between $G(n, p)$ and $G(n, M)$ can be read as follows.

Lemma 5.3 (cf. [2, p. 38] and [12, p. 17]) Let $\mathcal{Q}$ be any property of a group, and denote by $\mathbb{P}_{P}\left(G_{P} \in \mathcal{Q}\right)$ (resp. $\mathbb{P}_{P^{\prime \prime}}\left(G_{P} \in \mathcal{Q}\right)$ ) the probability of $G_{P}$ given by $P \in P\left(m, \eta, d, c_{0}\right)$ (resp. $P \in P^{\prime \prime}\left(m, \eta, d, c_{0}\right)$ ) having property $\mathcal{Q}$. Then we have

$$
\mathbb{P}_{P}\left(G_{P} \in \mathcal{Q}\right) \leq 3 \sqrt{c_{0}(2 m)^{d \eta}} \mathbb{P}_{P^{\prime \prime}}\left(G_{P} \in \mathcal{Q}\right) \text {. }
$$

Proof Set $N=(2 m)^{\eta}, M=c_{0}(2 m)^{d \eta}, p=\left(c_{0}(2 m)^{d \eta}\right) /(2 m)^{\eta}$ and $q=1-p$. By the law of total probability, we have

$$
\begin{aligned}
\mathbb{P}_{P^{\prime \prime}}\left(G_{P} \in \mathcal{Q}\right) & =\sum_{s=0}^{N} \mathbb{P}_{P, s}\left(G_{P} \in \mathcal{Q}\right){ }_{N} C_{s} p^{s} q^{N-s} \\
& \geq \mathbb{P}_{P, M}\left(G_{P} \in \mathcal{Q}\right)_{N} C_{M} p^{M} q^{N-M},
\end{aligned}
$$

where $P_{P, s}\left(G_{P} \in \mathcal{Q}\right)$ denotes the probability of $G_{P} \in \mathcal{Q}$ in the model $P\left(m, \eta, d, c_{0}^{\prime}\right)$ with $s=c_{0}^{\prime}(2 m)^{d \eta}$. Now [2, p. 4] gives

$$
{ }_{N} C_{M} p^{M} q^{N-M} \geq e^{-1 /(6 M)} \frac{1}{\sqrt{2 \pi}}(p q N)^{-1 / 2} .
$$

Noting $M=p N$ implies the desired inequality.

\section{Proofs of Proposition 2.1 and 3.7}

We first prove Proposition 3.7 and then proceed to Proposition 2.1.

\subsection{Proof of Proposition 3.7}

Let $c_{0}$ be a positive real number with $c^{-1} \leq c_{0} \leq c$, where $c$ is the constant fixed in order to define $P(m, \eta, d)$, and set

$$
P\left(m, \eta, d, c_{0}\right)=\left\{P=(S, R) \in P(m, \eta, d) \mid \# R=c_{0}(2 m)^{d \eta}\right\}
$$

as above. Recall that $\eta=2 n+l \geq(2 k+1) l$, take $d>k /(2 k+1)$, and set $\varepsilon_{0}=d(2 k+1)-k$. Because of $(2-1)$, we see that

$$
\begin{aligned}
d \eta & \geq d(2 k+1) l=\varepsilon_{0} l+k l, \\
d \eta-n & \geq d(2 k+1) l-(k l+(2 k+1))=\varepsilon_{0} l-(2 k+1), \\
d \eta-n & \leq d((2 k+1) l+2(2 k+1))-k l=\varepsilon_{0} l+2 d(2 k+1) .
\end{aligned}
$$


We will show that, for any $\varepsilon^{\prime}>0$, there exist constants $a_{1}$ and $a_{2}$ depending only on $m, k, c$, and $\varepsilon^{\prime}$ (and independent of $l$ and $c_{0}$ ) such that

$$
\frac{\left\{P \in P\left(m, \eta, d, c_{0}\right) \mid \begin{array}{c}
(3-6)^{\prime} \text { holds for } \forall Y, \forall \rho: \Gamma_{P} \longrightarrow \operatorname{Isom}(Y), \\
\forall \text { nonconstant } \rho \text {-equivariant map } f
\end{array}\right\}}{\# P\left(m, \eta, d, c_{0}\right)}
$$

holds for large $l$ (and hence for large $\eta$ ), where

$$
\left(1-\varepsilon^{\prime}\right) R Q^{G}(\bar{f}) \leq \frac{E_{\mu^{l}}(\tilde{f})}{E_{\mu^{2 n}}(\tilde{f})} \leq\left(1+\varepsilon^{\prime}\right) R Q^{G}(\bar{f}) .
$$

It is clear that this implies Proposition 3.7.

Since we are assuming $d<1 / 2$, we may deal with $P^{\prime}\left(m, \eta, d, c_{0}\right)$ instead of $P\left(m, \eta, d, c_{0}\right)$ as we have just explained in $\S 5.3$. Thus what we will actually show is that, for any $\varepsilon^{\prime}>0$, there exist constants $a_{1}$ and $a_{2}$ depending only on $m, k, c$, and $\varepsilon^{\prime}$ (and independent of $l$ and $c_{0}$ ) such that

$$
\begin{aligned}
& \mathbb{P}\left(\begin{array}{c}
P \in P^{\prime}\left(m, \eta, d, c_{0}\right) \text { satisfies }(3-6)^{\prime} \\
\text { for } \forall Y, \forall \rho: \Gamma_{P} \longrightarrow \operatorname{Isom}(Y), \\
\forall \text { nonconstant } \rho \text {-equivariant map } f
\end{array}\right) \\
\geq & 1-a_{1} \exp \left(-a_{2}(2 m)^{\varepsilon_{0} l}\right)
\end{aligned}
$$

holds for large $l$ (and hence for large $\eta$ ). Then this implies Proposition 3.7.

Let $w \in W_{\eta}$. We denote the word given by the first $n$ letters of $w$ by $w_{1}$, the word formed by the next $l$ letters by $u$, and the inverse word of the last $n$ letters by $w_{2}$; thus $w$ can be written as $w=w_{1} u w_{2}^{-1}$. Take $P=(S, R) \in P^{\prime}\left(m, \eta, d, c_{0}\right)$. Let $\rho: \Gamma_{P} \longrightarrow$ $\operatorname{Isom}(Y)$ be a homomorphism, and take any $\rho$-equivariant map $f: \Gamma_{P} \longrightarrow Y$. Then we can consider $\tilde{\rho}$-equivariant map $\tilde{f}: \Gamma \longrightarrow Y$, where $\tilde{\rho}=\rho \circ \pi_{P}: \Gamma \longrightarrow \operatorname{Isom}(Y)$. Regarding $w_{i}, i=1,2$, as an element $\pi_{P}\left(\overline{w_{i}}\right) \in \Gamma_{P}$ in a natural way, we get a map 
$\bar{f}: V \longrightarrow Y$ induced from $f$. Since $V=W_{n}$, we have

$$
\begin{aligned}
& \|d \bar{f}\|_{L^{2}}^{2}=\frac{1}{2} \sum_{v, v^{\prime} \in W_{n}} \nu\left(v, v^{\prime}\right) d_{Y}\left(\bar{f}(v), \bar{f}\left(v^{\prime}\right)\right)^{2} \\
& =\frac{1}{2} \sum_{v, v^{\prime} \in W_{n}} \nu\left(v, v^{\prime}\right) d_{Y}\left(\tilde{f}(\bar{v}), \tilde{f}\left(\overline{v^{\prime}}\right)\right)^{2} \\
& =\frac{1}{2} \sum_{v, v^{\prime} \in W_{n}} \nu\left(v, v^{\prime}\right) d_{Y}\left(\tilde{f}(e), \tilde{f}\left(\bar{v}^{-1} \overline{v^{\prime}}\right)\right)^{2} \\
& =\frac{1}{2} \sum_{\gamma \in \Gamma} \sum_{v, v^{\prime} \in W_{n}: \bar{v}^{-1} \bar{v}^{\prime}=\gamma} \nu\left(v, v^{\prime}\right) d_{Y}(\tilde{f}(e), \tilde{f}(\gamma))^{2}
\end{aligned}
$$

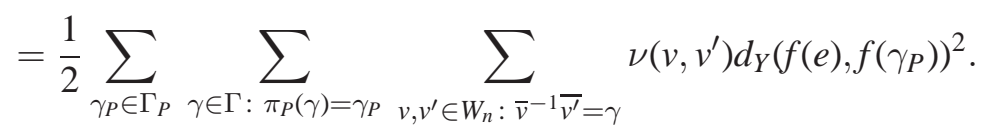

Note that $w=w_{1} u w_{2}^{-1} \in R$ means that $\pi_{P}\left(\overline{w_{1} u}\right)=\pi_{P}\left(\overline{w_{2}}\right)$ in $\Gamma_{P}$. Hence $\pi_{P}\left(\overline{w_{1}}-1 \overline{w_{2}}\right)=$ $\pi_{P}(\bar{u})$ and $\pi_{P}\left(\bar{w}_{2}^{-1} \overline{w_{1}}\right)=\pi_{P}\left(\bar{u}^{-1}\right)$. Thus, recalling the definition of $\nu\left(v, v^{\prime}\right)$ and fixing $\gamma_{P} \in \Gamma_{P}$, we can rewrite as

$$
\begin{aligned}
& \sum_{\gamma \in \Gamma: \pi_{P}(\gamma)=\gamma_{P}} \sum_{v, v^{\prime} \in W_{n}: \bar{v}^{-1} \bar{v}^{\prime}=\gamma} \nu\left(v, v^{\prime}\right) \\
& =\sum_{\gamma \in \Gamma: \pi_{P}(\gamma)=\gamma_{P}} \sum_{v, v^{\prime} \in W_{n}: \bar{v}^{-1} \overline{v^{\prime}}=\gamma} \frac{\#\left\{w \in R \mid w_{1}=v \text { and } w_{2}=v^{\prime}\right\}}{2 \# R} \\
& +\frac{\#\left\{w \in R \mid w_{1}=v^{\prime} \text { and } w_{2}=v\right\}}{2 \# R} \\
& =\frac{\#\left\{w \in R \mid \pi_{P}(\bar{u})=\gamma_{P} \text { or } \pi_{P}\left(\bar{u}^{-1}\right)=\gamma_{P}\right\}}{2 \# R} .
\end{aligned}
$$

Therefore we get

$$
\|d \bar{f}\|_{L^{2}}^{2}=\frac{1}{2} \sum_{\gamma_{P} \in \Gamma_{P}} \frac{\#\left\{w \in R \mid \pi_{P}(\bar{u})=\gamma_{P} \text { or } \gamma_{P}^{-1}\right\}}{2 \# R} d_{Y}\left(f(e), f\left(\gamma_{P}\right)\right)^{2}
$$

Since

$$
\#\left\{w \in R \mid \pi_{P}(\bar{u})=\gamma_{P} \text { or } \gamma_{P}^{-1}\right\}=\sum_{\gamma \in \Gamma: \pi_{P}(\gamma)=\gamma_{P}} \#\left\{w \in R \mid \bar{u}=\gamma \text { or } \gamma^{-1}\right\},
$$

lifting (6-3) to $\Gamma$ yields

$$
\|\bar{d}\|_{L^{2}}^{2}=\frac{1}{2} \sum_{\gamma \in \Gamma} \frac{\#\left\{w \in R \mid \bar{u}=\gamma \text { or } \gamma^{-1}\right\}}{2 \# R} d_{Y}(\tilde{f}(e), \tilde{f}(\gamma))^{2}
$$


Note that if $\bar{u}=e$, then $d_{Y}(\tilde{f}(e), \tilde{f}(\gamma))=d_{Y}(\tilde{f}(e), \tilde{f}(e))=0$, and hence we may ignore this case in what follows. Then we have

$$
\begin{aligned}
& \#\left\{w \in R \mid \bar{u}=\gamma \text { or } \gamma^{-1}\right\}=\#\{w \in R \mid \bar{u}=\gamma\}+\#\left\{w \in R \mid \bar{u}=\gamma^{-1}\right\} \\
= & \sum_{v \in W_{l}: \bar{v}=\gamma} \#\{w \in R \mid u=v\}+\sum_{v^{\prime} \in W_{l}: \bar{v}^{\prime}=\gamma^{-1}} \#\left\{w \in R \mid u=v^{\prime}\right\}
\end{aligned}
$$

for $\gamma \neq e$. Now let $X_{v, l}: P^{\prime}\left(m, \eta, d, c_{0}\right) \longrightarrow \mathbb{Z}$ be a random variable defined by

$$
X_{v, l}(P)=\#\{w \in R \mid u=v\},
$$

where $v \in W_{l}$. By the definition of $P^{\prime}\left(m, \eta, d, c_{0}\right), X_{v, l}$ has the binomial distribution $\operatorname{Bi}\left(\# R, \frac{1}{\# W_{l}}\right)$ with mean $\frac{\# R}{\# W_{l}}$. Note that, for $P=(S, R) \in P^{\prime}\left(m, \eta, d, c_{0}\right)$ under consideration, we can write as

$$
\begin{aligned}
\|d \bar{f}\|_{L^{2}}^{2}=\frac{1}{2} \sum_{\gamma \in \Gamma} & \frac{\sum_{v \in W_{l}: \bar{v}=\gamma} X_{v, l}(P)+\sum_{v^{\prime} \in W_{l}: \bar{v}^{\prime}=\gamma^{-1}} X_{v^{\prime}, l}(P)}{2 \# R} \\
& \times d_{Y}(\tilde{f}(e), \tilde{f}(\gamma))^{2} .
\end{aligned}
$$

Then, since $\mu^{l}(e, \gamma)=\#\left\{w \in W_{l} \mid \bar{w}=\gamma\right\} / \# W_{l}$, the expected value of the coefficient of $d_{Y}(\tilde{f}(e), \tilde{f}(\gamma))$ in (6-4) becomes

$$
\begin{aligned}
& \mathbb{E}\left(\frac{\sum_{v \in W_{l}: \bar{v}=\gamma} X_{v, l}+\sum_{v^{\prime} \in W_{l}: \overline{v^{\prime}}=\gamma^{-1}} X_{v^{\prime}, l}}{2 \# R}\right) \\
= & \frac{1}{2 \# R}\left(\sum_{v \in W_{l}: \bar{v}=\gamma} \mathbb{E}\left(X_{v, l}\right)+\sum_{v^{\prime} \in W_{l}: \bar{v}^{\prime}=\gamma^{-1}} \mathbb{E}\left(X_{v^{\prime}, l}\right)\right) \\
= & \frac{1}{2 \# R}\left(\sum_{v \in W_{l}: \bar{v}=\gamma} \frac{\# R}{\# W_{l}}+\sum_{v^{\prime} \in W_{l}: \bar{v}^{\prime}=\gamma^{-1}} \frac{\# R}{\# W_{l}}\right) \\
= & \frac{1}{2}\left(\mu^{l}(e, \gamma)+\mu^{l}\left(e, \gamma^{-1}\right)\right) \\
= & \mu^{l}(e, \gamma) .
\end{aligned}
$$

Therefore, if $X_{v, l}(P)=\#\{w \in R \mid u=v\}$ of $P=(S, R)$ is close to the expected value for all $v \in W_{l}$, then $\|d \bar{f}\|_{L^{2}}^{2}$ must be close to

$$
\frac{1}{2} \sum_{\gamma \in \Gamma} \mu^{l}(e, \gamma) d_{Y}(\tilde{f}(e), \tilde{f}(\gamma))^{2}=E_{\mu^{l}}(\tilde{f}) .
$$

More precisely speaking, if $P=(S, R)$ satisfies

$$
(1-\varepsilon) \frac{\# R}{\# W_{l}} \leq X_{v, l}(P) \leq(1+\varepsilon) \frac{\# R}{\# W_{l}}
$$


for all $v \in W_{l}$, then we see that, for any $Y, \rho: \Gamma_{P} \longrightarrow \operatorname{Isom}(Y)$ and $\rho$-equivariant map $f$,

$$
(1-\varepsilon) E_{\mu^{l}}(\tilde{f}) \leq\|d \bar{f}\|_{L^{2}}^{2} \leq(1+\varepsilon) E_{\mu^{l}}(\tilde{f})
$$

holds.

On the other hand, we have

$$
\begin{aligned}
& F(\bar{f})=\frac{1}{2} \sum_{v, v^{\prime} \in W_{n}} \nu(v) \nu\left(v^{\prime}\right) d_{Y}\left(\bar{f}(v), \bar{f}\left(v^{\prime}\right)\right)^{2} \\
& =\frac{1}{2} \sum_{v, v^{\prime} \in W_{n}} \nu(v) \nu\left(v^{\prime}\right) d_{Y}\left(f\left(\pi_{P}(\bar{v})\right), f\left(\pi_{P}\left(\overline{v^{\prime}}\right)\right)\right)^{2} \\
& =\frac{1}{2} \sum_{v, v^{\prime} \in W_{n}} \nu(v) \nu\left(v^{\prime}\right) d_{Y}\left(f(e), f\left(\pi_{P}\left(\bar{v}^{-1} \overline{v^{\prime}}\right)\right)\right)^{2} \\
& =\frac{1}{2} \sum_{\gamma, \gamma^{\prime} \in \Gamma} \sum_{v, v^{\prime} \in W_{n}: \bar{v}=\gamma, \overline{v^{\prime}}=\gamma^{\prime}} \nu(v) \nu\left(v^{\prime}\right) d_{Y}\left(\tilde{f}(e), \tilde{f}\left(\gamma^{-1} \gamma^{\prime}\right)\right)^{2} .
\end{aligned}
$$

Let $X_{i, v, n}: P\left(m, \eta, d, c_{0}\right) \longrightarrow \mathbb{Z}, i=1,2$, be a random variable defined by

$$
X_{i, v, n}(P)=\#\left\{w \in R \mid w_{i}=v\right\},
$$

where $v \in W_{n}$. Using this random variable and recalling the definition of $\nu(v)$, we see that, for $P=(S, R)$,

$$
\begin{aligned}
\nu(v) & =\sum_{v^{\prime} \in W_{n}} \nu\left(v, v^{\prime}\right)=\frac{\#\left\{w \in R \mid w_{1}=v\right\}+\#\left\{w \in R \mid w_{2}=v\right\}}{2 \# R} \\
& =\frac{X_{1, v, n}(P)+X_{2, v, n}(P)}{2 \# R}
\end{aligned}
$$

holds. Therefore we get

$$
\begin{aligned}
F(\bar{f})=\frac{1}{2} \sum_{\gamma, \gamma^{\prime} \in \Gamma} \frac{\sum_{v \in W_{n}: \bar{v}=\gamma}\left(X_{1, v, n}(P)+X_{2, v, n}(P)\right)}{2 \# R} \\
\times \frac{\sum_{v \in W_{n}: \bar{v}=\gamma^{\prime}}\left(X_{1, v, n}(P)+X_{2, v, n}(P)\right)}{2 \# R} \\
\times d_{Y}\left(\tilde{f}(e), \tilde{f}\left(\gamma^{-1} \gamma^{\prime}\right)\right)^{2} .
\end{aligned}
$$


Since $X_{i, v, n}$ has the binomial distribution $\mathrm{Bi}\left(\# R, \frac{1}{\# W_{n}}\right)$ for $i=1,2$, we get

$$
\begin{aligned}
\mathbb{E}\left(\frac{\sum_{v \in W_{n}: \bar{v}=\gamma} X_{1, v, n}+X_{2, v, n}}{2 \# R}\right) & =\frac{1}{2 \# R} \sum_{v \in W_{n}: \bar{v}=\gamma}\left(\mathbb{E}\left(X_{1, v, n}\right)+\mathbb{E}\left(X_{2, v, n}\right)\right) \\
& =\frac{1}{\# R} \#\left\{v \in W_{n} \mid \bar{v}=\gamma\right\} \frac{\# R}{\# W_{n}} \\
& =\mu^{n}(e, \gamma) .
\end{aligned}
$$

Therefore, if both $\#\left\{w \in R \mid w_{1}=v\right\}$ and $\#\left\{w \in R \mid w_{2}=v\right\}$ are close to the expected value for all $v \in W_{n}$, then $F(\bar{f})$ must be close to

$$
\begin{aligned}
& \frac{1}{2} \sum_{\gamma, \gamma^{\prime} \in \Gamma} \mu^{n}(e, \gamma) \mu^{n}\left(e, \gamma^{\prime}\right) d_{Y}\left(\tilde{f}(e), \tilde{f}\left(\gamma^{-1} \gamma^{\prime}\right)\right)^{2} \\
= & \frac{1}{2} \sum_{\gamma, \gamma^{\prime} \in \Gamma} \mu^{n}\left(\gamma^{-1}, e\right) \mu^{n}\left(\gamma^{-1}, \gamma^{-1} \gamma^{\prime}\right) d_{Y}\left(\tilde{f}(e), \tilde{f}\left(\gamma^{-1} \gamma^{\prime}\right)\right)^{2} \\
= & \frac{1}{2} \sum_{\gamma} \mu^{n}\left(\gamma^{-1}, e\right) \sum_{\gamma^{\prime}} \mu^{n}\left(\gamma^{-1}, \gamma^{-1} \gamma^{\prime}\right) d_{Y}\left(\tilde{f}(e), \tilde{f}\left(\gamma^{-1} \gamma^{\prime}\right)\right)^{2} \\
= & \frac{1}{2} \sum_{\gamma} \mu^{n}\left(e, \gamma^{-1}\right) \sum_{\gamma^{\prime \prime}} \mu^{n}\left(\gamma^{-1}, \gamma^{\prime \prime}\right) d_{Y}\left(\tilde{f}(e), \tilde{f}\left(\gamma^{\prime \prime}\right)\right)^{2} \\
= & \frac{1}{2} \sum_{\gamma^{\prime \prime}} \sum_{\gamma} \mu^{n}\left(e, \gamma^{-1}\right) \mu^{n}\left(\gamma^{-1}, \gamma^{\prime \prime}\right) d_{Y}\left(\tilde{f}(e), \tilde{f}\left(\gamma^{\prime \prime}\right)\right)^{2} \\
= & \frac{1}{2} \sum_{\gamma^{\prime \prime}} \mu^{2 n}\left(e, \gamma^{\prime \prime}\right) d_{Y}\left(\tilde{f}(e), \tilde{f}\left(\gamma^{\prime \prime}\right)\right)^{2} \\
= & E_{\mu^{2 n}}(\tilde{f}) .
\end{aligned}
$$

Actually, if $P=(S, R)$ satisfies

$$
(1-\varepsilon) \frac{\# R}{\# W_{n}} \leq X_{i, v, n}(P) \leq(1+\varepsilon) \frac{\# R}{\# W_{n}}, \quad i=1,2
$$

for all $v \in W_{n}$, then we see that, for any $Y, \rho: \Gamma_{P} \longrightarrow \operatorname{Isom}(Y)$, and $\rho$-equivariant $\operatorname{map} f$,

$$
(1-\varepsilon)^{2} E_{\mu^{2 n}}(\tilde{f}) \leq F(\bar{f}) \leq(1+\varepsilon)^{2} E_{\mu^{2 n}}(\tilde{f})
$$

holds.

Since our random variables $X_{v, l}$ and $X_{i, v, n}$ are binomially distributed with distribution 
$\operatorname{Bi}\left(\# R, \frac{1}{\# W_{l}}\right)$ and $\mathrm{Bi}\left(\# R, \frac{1}{\# W_{n}}\right)$ respectively, we have

$$
\begin{aligned}
\mathbb{E}\left(X_{v, l}\right) & =\frac{\# R}{\# W_{l}}=c_{0}(2 m)^{d \eta-l} \geq c_{0}(2 m)^{\varepsilon_{0} l+(k-1) l} \\
\mathbb{E}\left(X_{i, v, n}\right) & =\frac{\# R}{\# W_{n}}=c_{0}(2 m)^{d \eta-n} \geq c_{0}(2 m)^{\varepsilon_{0} l-(2 k+1)}
\end{aligned}
$$

by (2-1) and (6-1). Since each expected value does not depend on the choice of $v$, we denote it by $\mu_{l}$ and $\mu_{i, n}$ respectively. Using (5-2), we see that, for any given $\varepsilon>0$,

$$
\begin{aligned}
& \mathbb{P}\left(\left|X_{v, l}-\mu_{l}\right|>\varepsilon \mu_{l}\right) \leq 2 \exp \left(-\frac{\varepsilon^{2} c_{0}(2 m)^{\left(\varepsilon_{0}+k-1\right) l}}{3}\right), \\
& \mathbb{P}\left(\left|X_{i, v, n}-\mu_{i, n}\right|>\varepsilon \mu_{i, n}\right) \leq 2 \exp \left(-\frac{\varepsilon^{2} c_{0}(2 m)^{\varepsilon_{0} l-(2 k+1)}}{3}\right)
\end{aligned}
$$

hold. Since $\# W_{n}=(2 m)^{n}, n \leq k l+(2 k+1)$, and $d(2 k+1)=\varepsilon_{0}+k$, we have

$$
\begin{aligned}
& \mathbb{P}\left(\left|X_{i, v, n}-\mu_{i, n}\right|>\varepsilon \mu_{i, n} \text { for some } v \in W_{n}\right) \\
\leq & (2 m)^{n} 2 \exp \left(-\frac{\varepsilon^{2} c_{0}(2 m)^{\varepsilon_{0} l-(2 k+1)}}{3}\right) \\
\leq & 2 \max _{l}\left\{(2 m)^{k l+(2 k+1)} \exp \left(-\frac{\varepsilon^{2} c_{0}(2 m)^{\varepsilon_{0} l-(2 k+1)}}{6}\right)\right\} \\
& \times \exp \left(-\frac{\varepsilon^{2} c_{0}(2 m)^{\varepsilon_{0} l-(2 k+1)}}{6}\right) .
\end{aligned}
$$

Letting

$$
\begin{aligned}
& a=2 \max _{l}\left\{(2 m)^{k l+(2 k+1)} \exp \left(-\frac{\varepsilon^{2} c^{-1}(2 m)^{\varepsilon_{0} l-(2 k+1)}}{6}\right)\right\}, \\
& a^{\prime}=\frac{\varepsilon^{2} c^{-1}(2 m)^{-(2 k+1)}}{6},
\end{aligned}
$$

we obtain

$$
\mathbb{P}\left(\left|X_{i, v, n}-\mu_{i, n}\right|>\varepsilon \mu_{i, n} \text { for some } v \in W_{n}\right) \leq a \exp \left(-a^{\prime}(2 m)^{\varepsilon_{0} l}\right)
$$

for any $P^{\prime}\left(m, \eta, d, c_{0}\right)$ for $c^{-1} \leq c_{0} \leq c$. Since $k \geq 1$,

$$
\mathbb{P}\left(\left|X_{v, l}-\mu_{l}\right|>\varepsilon \mu_{l} \text { for some } v \in W_{l}\right) \leq a \exp \left(-a^{\prime}(2 m)^{\varepsilon_{0} l}\right)
$$

holds for the same $a$ and $a^{\prime}$ as above. Recalling (6-5) and (6-6) and letting $a_{1}=3 a$ and $a_{2}=a^{\prime}$, we see that these inequalities imply

$$
\mathbb{P}\left(\begin{array}{c}
P \in P^{\prime}\left(m, \eta, d, c_{0}\right) \text { satisfies (6-7) } \\
\text { for } \forall Y, \forall \rho: \Gamma_{P} \longrightarrow \operatorname{Isom}(Y), \\
\forall \text { nonconstant } \rho \text {-equivariant map } f
\end{array}\right) \geq 1-a_{1} \exp \left(-a_{2}(2 m)^{\varepsilon_{0} l}\right)
$$


where

$$
\frac{1-\varepsilon}{(1+\varepsilon)^{2}} R Q^{G}(\bar{f}) \leq \frac{E_{\mu^{\prime}}(\tilde{f})}{E_{\mu^{2 n}}(\tilde{f})} \leq \frac{1+\varepsilon}{(1-\varepsilon)^{2}} R Q^{G}(\bar{f}) .
$$

Thus, by taking $\varepsilon$ suitably, this implies (6-2). This completes the proof of Proposition 3.7 .

Remark 2 We should mention that it is also possible to prove Proposition 3.7 using the model $P^{\prime \prime}\left(m, \eta, d, c_{0}\right)$ introduced in $\S 5.3$ with the help of Lemma 5.3; thus we can drop the assumption $d<1 / 2$ in Proposition 3.7. However, the appearance of $\mu^{l}$ and $\mu^{n}$ in the proof becomes less natural compared with the proof presented above.

\subsection{Proof of Proposition 2.1}

Our proof follows the idea of [4], although our model is different from theirs and a certain modification is needed.

As we have mentioned in $\S 5.3$, we will deal with $P^{\prime \prime}\left(m, \eta, d, c_{0}\right)$ in order to prove Proposition 2.1. Let $P \in P^{\prime \prime}\left(m, \eta, d, c_{0}\right)$ and $G_{P}=\left(V, E_{P}\right)$ the graph associated to $P$. Recall that $G_{P}$ has measures on $V \times V$ and $V$ both denoted by $\nu$, which are used to define the inner product on the space of functions on $V$ and the Laplacian $\Delta_{P}$. We are going to deal with the eigenvalues of $\Delta_{P}$, and it is convenient to give an expression of $\Delta_{P}$ by matrices. Let us denote $V=\left\{v_{1}, \ldots, v_{(2 m)^{n}}\right\}$, and $\mathbf{e}_{i}$ be a function defined by

$$
\mathbf{e}_{i}\left(v_{j}\right)= \begin{cases}\frac{1}{\sqrt{\nu\left(v_{i}\right)}} & \text { if } i=j \\ 0 & \text { if } i \neq j\end{cases}
$$

Then $\left\{\mathbf{e}_{1}, \ldots, \mathbf{e}_{(2 m)^{n}}\right\}$ forms an orthonormal basis of the space of functions on $V$ with respect to the inner product $(\cdot, \cdot)_{L^{2}}$. Note that

$$
\Delta_{P} \mathbf{e}_{i}\left(v_{j}\right)=\mathbf{e}_{i}\left(v_{j}\right)-\sum_{v^{\prime} \in V} \frac{\nu\left(v_{j}, v^{\prime}\right)}{\nu\left(v_{j}\right)} \mathbf{e}_{i}\left(v^{\prime}\right)=\frac{\delta_{i j}}{\sqrt{\nu\left(v_{i}\right)}}-\frac{\nu\left(v_{j}, v_{i}\right)}{\nu\left(v_{j}\right) \sqrt{\nu\left(v_{i}\right)}},
$$

therefore

$$
\Delta_{P} \mathbf{e}_{i}=\mathbf{e}_{i}-\sum_{j} \frac{\nu\left(v_{j}, v_{i}\right)}{\sqrt{\nu\left(v_{j}\right) \nu\left(v_{i}\right)}} \mathbf{e}_{j},
$$

and $\Delta_{P}$ can be expressed as

$$
\Delta_{P}=I-A^{\prime}
$$


where $I$ denotes the identity matrix, and $A^{\prime}$ is expressed by means of its entries as

$$
A^{\prime}=\left(\frac{\nu\left(v_{i}, v_{j}\right)}{\sqrt{\nu\left(v_{i}\right) \nu\left(v_{j}\right)}}\right) \text {. }
$$

Note that, by definition,

$$
\frac{\nu\left(v_{i}, v_{j}\right)}{\sqrt{\nu\left(v_{i}\right) \nu\left(v_{j}\right)}}=\frac{\text { multiplicity of }\left\{v_{i}, v_{j}\right\}}{\sqrt{\operatorname{deg}\left(v_{i}\right) \operatorname{deg}\left(v_{j}\right)}} .
$$

Let $A$ be the multiplicity matrix (the adjacency matrix taking the multipicity in account) and $D$ the square root of the inverse of the degree matrix:

$$
A=\left(\text { multiplicity of }\left\{v_{i}, v_{j}\right\}\right) \quad \text { and } \quad D=\left(\frac{\delta_{i j}}{\sqrt{\operatorname{deg}\left(v_{i}\right)}}\right) \text {. }
$$

Then we see that $A^{\prime}=D A D$.

Let $\pi_{0}$ be the orthogonal projection onto the space of constant functions on $V$. Note that

$$
M=\left(I-\Delta_{P}\right)\left(I-\pi_{0}\right)=I-\Delta_{P}-\pi_{0}=D A D-\pi_{0}
$$

has eigenvalues 0 and $1-\lambda_{i}\left(G_{P}\right), i \neq 0$, where $0=\lambda_{0}\left(G_{P}\right) \leq \lambda_{1}\left(G_{P}\right) \leq \ldots \lambda_{m}\left(G_{P}\right)$ are the eigenvalues of $\Delta_{P}$, and eigenfunctions corresponding to 0 are constant functions. (Here we regard $M$ is given as a matrix form with respect the orthonormal basis $\left\{\mathbf{e}_{i}\right\}$. Note that the eigenfunctions of $M$ are the same as that of $\Delta_{P}$.) Thus we have

$$
\max _{i \neq 0}\left|1-\lambda_{i}\left(G_{P}\right)\right| \leq\|M\|,
$$

where $\|M\|$ denotes the operator norm of $M$.

We regard $\|M\|=\left\|M_{P}\right\|$ as a random variable on $P^{\prime \prime}\left(m, \eta, d, c_{0}\right)$ and will show that, for any $\varepsilon^{\prime}>0$, there exist positive constants $a_{1}$ and $a_{2}$ depending only on $m, k, c$, and $\varepsilon^{\prime}$ (and, in particular, independent of $c_{0}$ ) and $a_{3}$ depending on $m, k, \varepsilon^{\prime}$, and $\alpha$ such that

$$
\mathbb{P}_{P^{\prime \prime}}\left(\|M\|>\varepsilon^{\prime}\right) \leq a_{1} \exp \left(-a_{2}(2 m)^{\varepsilon_{0} l}\right)+a_{3}(2 m)^{-\left(\alpha \varepsilon_{0}-k\right) l}
$$

holds, where $\alpha$ is an arbitrary natural number and $\varepsilon_{0}=d(2 k+1)-k$. Once (6-9) is proven, recalling Lemma 5.3 and $d \eta \leq\left(k+\varepsilon_{0}\right) l+2\left(k+\varepsilon_{0}\right)$, we obtain

$$
\begin{aligned}
& \mathbb{P}_{P}\left(\|M\|>\varepsilon^{\prime}\right) \\
\leq & 3 \sqrt{c_{0}(2 m)^{d \eta}} \mathbb{P}_{P^{\prime \prime}}\left(\|M\|>\varepsilon^{\prime}\right) \\
\leq & 3 c^{1 / 2}(2 m)^{\left(k+\varepsilon_{0}\right) l+2\left(k+\varepsilon_{0}\right)}\left(a_{1} \exp \left(-a_{2}(2 m)^{\varepsilon_{0} l}\right)+a_{3}(2 m)^{-\left(\alpha \varepsilon_{0}-k\right) l}\right) \\
\leq & a_{1}^{\prime}(2 m)^{\left(k+\varepsilon_{0}\right) l} \exp \left(-a_{2}(2 m)^{\varepsilon_{0} l}\right)+a_{3}^{\prime}(2 m)^{-\left(\alpha \varepsilon_{0}-\left(2 k+\varepsilon_{0}\right)\right) l} .
\end{aligned}
$$


By choosing $\alpha>\left(2 k+\varepsilon_{0}\right) / \varepsilon_{0}$ and setting $\alpha_{0}=\alpha-\left(2 k+\varepsilon_{0}\right) / \varepsilon_{0}$, we see that

$$
\mathbb{P}_{P}\left(\|M\| \leq \varepsilon^{\prime}\right) \geq 1-a_{1}^{\prime}(2 m)^{\left(k+\varepsilon_{0}\right) l} \exp \left(-a_{2}(2 m)^{\varepsilon_{0} l}\right)-a_{3}^{\prime \prime}(2 m)^{-\left(\alpha_{0} \varepsilon_{0}\right) l} \rightarrow 1
$$

as $\eta \rightarrow \infty$, where $a_{1}^{\prime}, a_{3}^{\prime \prime}$, and $\alpha_{0}$ depends only on $m, k, c, \varepsilon^{\prime}$, and $\alpha$ chosen as above (and independent of $c_{0}$ ). Together with (6-8), this implies Proposition 2.1. In what follows we will deal only with $P^{\prime \prime}\left(m, \eta, d, c_{0}\right)$, and will drop subscript $P^{\prime \prime}$ of $\mathbb{P}_{P^{\prime \prime}}$, since there will be no chance of confusion.

The first step of the proof of (6-9) is to estimate $\|B\|$ of an operator $B$ defined below, which approximates $M$ with high probability. Recall our random graph has $\# V=(2 m)^{n}$ vertices and, for each pair of vertices, the multiplicity of the edge joining them is at most $(2 m)^{l}=\# W_{l}$. Since each word in $W_{\eta}$ is chosen with probability $\left(c_{0}(2 m)^{d \eta}\right) /(2 m)^{\eta}$, the multiplicity of an edge has the binomial distribution $\operatorname{Bi}\left((2 m)^{l},\left(c_{0}(2 m)^{d \eta}\right) /(2 m)^{\eta}\right)$, and the expected multiplicity $\mu_{e}$ of each edge is $(2 m)^{l} \times\left(c_{0}(2 m)^{d \eta}\right) /(2 m)^{\eta}$. Thus, by recalling (2-1) and (6-1), the expected multiplicity $\mu_{e}$ can be estimated as

$$
\begin{aligned}
\mu_{e} & =c_{0}(2 m)^{l+(d-1)(2 n+l)} \\
& \leq 2 c_{0}(2 m)^{d(2 k+1) l+2 d(2 k+1)-(2 k+1) l+l} \\
& =2 c_{0}(2 m)^{-\left(k-\varepsilon_{0}\right) l+2 d(2 k+1)} .
\end{aligned}
$$

On the other hand, since a vertex is given as the first (or the last) $n$ letters of elements of $W_{\eta}$, it is followed by (or follows) the elements of $W_{n+l}$. Therefore, for any $v \in W_{n}$, the random variable $Y_{i, v, n}$ on $P^{\prime \prime}\left(m, \eta, d, c_{0}\right)$ defined by

$$
Y_{i, v, n}(P)=\#\left\{w \in R \mid w_{i}=v\right\}
$$

has the binomial distribution $\operatorname{Bi}\left((2 m)^{n+l},\left(c_{0}(2 m)^{d \eta}\right) /(2 m)^{\eta}\right)$. Since the degree of a vertex $v$ is given by $\operatorname{deg}(v)=Y_{1, v, n}+Y_{2, v, n}$, the expected degree $\mu_{v}$ of a vertex is given by $\mu_{v}=2(2 m)^{n+l} \times\left(c_{0}(2 m)^{d \eta}\right) /(2 m)^{\eta}$. Thus, again by (2-1) and (6-1), $\mu_{v}$ can be estimated as

$$
\mu_{v}=2 c_{0}(2 m)^{d \eta}(2 m)^{-n} \geq 2 c_{0}(2 m)^{\varepsilon_{0} l-(2 k+1)} .
$$

Let a matrix $B$ be

$$
B=\frac{1}{\mu_{v}}\left(A-\mu_{e} K\right),
$$

where $K$ is a matrix all of whose entries are 1. Note that each entries of the matrix $A-\mu_{e} K$ is equal to

$$
\text { (multiplicity of } \left.\left\{v_{i}, v_{j}\right\}\right)-\mu_{e},
$$

and hence, writing $B=\left(b_{i j}\right)$, we see that $\mathbb{E}\left(b_{i j}\right)=0$ for each $(i, j)$. We first show that the eigenvalue of $B$ approaches to 0 as $l \rightarrow \infty$. Note that, since $B$ is symmetric

$$
\operatorname{trace}\left(B^{2 \alpha}\right)=\lambda_{1}(B)^{2 \alpha}+\ldots \lambda_{(2 m)^{n}}(B)^{2 \alpha}
$$


for any positive integer $\alpha$, where $\lambda_{i}(B)$ 's are eigenvalues of $B$ (counted with multiplicity). Let $\lambda_{1}(B)$ be the eigenvalue of $B$ with maximum absolute value, which is equal to the operator norm of $B:\|B\|=\left|\lambda_{1}(B)\right|$. Then we have

$$
\mathbb{E}\left(\|B\|^{2 \alpha}\right)=\mathbb{E}\left(\lambda_{1}(B)^{2 \alpha}\right) \leq \mathbb{E}\left(\operatorname{trace}\left(B^{2 \alpha}\right)\right) .
$$

The $i$ th diagonal entry of $B^{2 \alpha}$ is a sum of terms of the form $b_{i i_{1}} b_{i_{1} i_{2}} \ldots b_{i_{2 \alpha-1} i}$, which corresponds to a closed path of length $2 \alpha$ in the complete graph (with self-loops) having $V$ as the vertex set with basepoint $v_{i}$.

Note that $b_{i j}$ 's are independent random variables, and that the mean of $b_{i j}$ is 0 as we have mentioned above. Therefore the expected value of the diagonal entries $B^{2 \alpha}$ involves only closed paths of length $2 \alpha$ all of whose edges are visited at least twice. Such a closed path is called a closed good walk in [7]. Now we see that $\mathbb{E}\left(\operatorname{trace}\left(B^{2 \alpha}\right)\right)$ is a sum of terms of the form

$$
\mathbb{E}\left(\prod b_{i j}^{\alpha_{i j}}\right)=\prod \mathbb{E}\left(b_{i j}^{\alpha_{i j}}\right),
$$

where $\alpha_{i j}$ 's are positive integers greater than 1 and their sum is equal to $2 \alpha$. The multiplicity of each edge is distributed binomially with mean $\mu_{e}$ and, by taking $l$ suitably large, we may assume $\mu_{e} \leq 2 c_{0}(2 m)^{-\left(k-\varepsilon_{0}\right) l+2 d(2 k+1)}<1$. Therefore, we can apply Lemma 5.2 to obtain

$$
\mathbb{E}\left(b_{i j}^{\alpha_{i j}}\right)=\frac{1}{\mu_{v}^{\alpha_{i j}}} \mathbb{E}\left(\left(\text { multiplicity of }\left\{v_{i}, v_{j}\right\}-\mu_{e}\right)^{\alpha_{i j}}\right) \leq c\left(\alpha_{i j}\right) \frac{1}{\mu_{v}^{\alpha_{i j}}} \mu_{e} .
$$

Let $W(\alpha, \beta)$ be the set of all closed good walks of length $2 \alpha$ which pass through $\beta+1$ distinct vertices. If $b_{i i_{1}} b_{i_{1} i_{2}} \ldots b_{i_{2 \alpha-1} i}$ corresponds to a walk in $W(\alpha, \beta)$, then there appear at least $\beta$ distinct $b_{i j}$ 's. Since we may assume $\mu_{e}<1$, we get

$$
\begin{aligned}
\prod \mathbb{E}\left(b_{i j}^{\alpha_{i j}}\right) & \leq c(\alpha) \frac{1}{\mu_{v}^{2 \alpha}} \mu_{e}^{\beta} \\
& \leq 2^{-2 \alpha} c(\alpha) c_{0}^{\beta-2 \alpha}(2 m)^{-2 \alpha \varepsilon_{0} l+2 \alpha(2 k+1)}(2 m)^{-\beta\left(k-\varepsilon_{0}\right) l+2 d \beta(2 k+1)}
\end{aligned}
$$

for some constant $c(\alpha)$. Note that

$$
\begin{aligned}
& \# W(\alpha, \beta) \\
\leq & \#(\text { choice of } \beta+1 \text { vertices from } V) \\
& \times \#(\text { good walks with length } 2 \alpha \text { in the complete graph with } \beta+1 \text { vertices }) \\
\leq & \# V^{\beta+1} c(\alpha, \beta)=(2 m)^{(\beta+1) n} c(\alpha, \beta) \leq c(\alpha, \beta)(2 m)^{(\beta+1)(k l+(2 k+1))}
\end{aligned}
$$

holds, where, clearly, $c(\alpha, \beta)$ is a constant depending only on $\alpha$ and $\beta$. (See [7] for a sharp bound of $W(\alpha, \beta)$.) 
Thus, noting $c^{-1} \leq c_{0} \leq c$ and summing up (6-10) over all good walks of length $2 \alpha$ gives

$$
\begin{aligned}
& \mathbb{E}\left(\operatorname{trace}\left(B^{2 \alpha}\right)\right) \\
& \leq 2^{-2 \alpha} c(\alpha) \sum_{\beta=0}^{\alpha} c^{2 \alpha-\beta} \# W(\alpha, \beta)(2 m)^{-2 \alpha \varepsilon_{0} l}(2 m)^{-\beta\left(k-\varepsilon_{0}\right) l}(2 m)^{(2 \alpha+2 d \beta)(2 k+1)} \\
& \leq\left(\frac{c}{2}\right)^{2 \alpha} c(\alpha) \sum_{\beta=0}^{\alpha} c(\alpha, \beta)(2 m)^{(\beta+1)(k l+(2 k+1))}(2 m)^{(2 \alpha+2 d \beta)(2 k+1)} \\
& \quad \times(2 m)^{-2 \alpha \varepsilon_{0} l-\beta\left(k-\varepsilon_{0}\right) l} \\
& \leq\left(\frac{c}{2}\right)^{2 \alpha} c(\alpha) \sum_{\beta=0}^{\alpha} c(\alpha, \beta)(2 m)^{(\beta+1+2 \alpha+2 d \beta)(2 k+1)}(2 m)^{(\beta+1) k l-2 \alpha \varepsilon_{0} l-\beta\left(k-\varepsilon_{0}\right) l} \\
& \leq\left(\frac{c}{2}\right)^{2 \alpha} c(\alpha)(2 m)^{(5 \alpha+1)(2 k+1)} \sum_{\beta=0}^{\alpha} c(\alpha, \beta)(2 m)^{k l-(2 \alpha-\beta) \varepsilon_{0} l}
\end{aligned}
$$

where we have used $\beta \leq \alpha$ to deduce the last inequality. By $\beta \leq \alpha$ again, and by setting $c^{\prime}(\alpha)=2^{-2 \alpha} c(\alpha) c^{2 \alpha}(2 m)^{(5 \alpha+1)(2 k+1)} \sum_{\beta} c(\alpha, \beta)$, we get

$$
\mathbb{E}\left(\operatorname{trace}\left(B^{2 \alpha}\right)\right) \leq c^{\prime}(\alpha)(2 m)^{k l-\alpha \varepsilon_{0} l} .
$$

Thus, by Markov's inequality (5-1), for any $\varepsilon>0$, we get

$$
\mathbb{P}\left(\|B\|^{2 \alpha} \geq \varepsilon^{2 \alpha}\right) \leq \frac{\mathbb{E}\left(\|B\|^{2 \alpha}\right)}{\varepsilon^{2 \alpha}} \leq \frac{\mathbb{E}\left(\operatorname{trace}\left(B^{2 \alpha}\right)\right)}{\varepsilon^{2 \alpha}}=\frac{c^{\prime}(\alpha)}{\varepsilon^{2 \alpha}}(2 m)^{k l-\alpha \varepsilon_{0} l},
$$

and hence

$$
\mathbb{P}(\|B\| \geq \varepsilon) \leq \frac{c^{\prime}(\alpha)}{\varepsilon^{2 \alpha}}(2 m)^{-\left(\alpha \varepsilon_{0}-k\right) l}
$$

Next we will show that $B$ actually approximates $M$ with high probability. Now take a matrix $M^{\prime}$ to be

$$
M^{\prime}=D\left(A-\mu_{e} K\right) D
$$

Writing $M^{\prime}=\left(m_{i j}^{\prime}\right)$, we see that

$$
m_{i j}^{\prime}=\frac{\mu_{v}}{\sqrt{\operatorname{deg}\left(v_{i}\right) \operatorname{deg}\left(v_{j}\right)}} b_{i j} .
$$


Let $f: V \longrightarrow \mathbb{R}$ and write $f=\sum_{i} f_{i} \mathbf{e}_{i}$. Since $M^{\prime}$ is symmetric,

$$
\begin{aligned}
\left\|M^{\prime}\right\| & =\max _{|f|=1}\left|\left(f, M^{\prime} f\right)\right| \\
& =\max _{|f|=1}\left|\sum_{i} f_{i}\left(\sum_{j} \frac{\mu_{v}}{\sqrt{\operatorname{deg}\left(v_{i}\right) \operatorname{deg}\left(v_{j}\right)}} b_{i j} f_{j}\right)\right| \\
& =\max _{|f|=1}\left|\sum_{i} \frac{\sqrt{\mu_{v}}}{\sqrt{\operatorname{deg}\left(v_{i}\right)}} f_{i}\left(\sum_{j} b_{i j} \frac{\sqrt{\mu_{v}}}{\sqrt{\operatorname{deg}\left(v_{j}\right)}} f_{j}\right)\right| \\
& =\max _{|f|=1}\left|\left(f^{\prime}, B f^{\prime}\right)\right| \\
& \leq\left.\max _{|f|=1}|| B|| f^{\prime}\right|^{2},
\end{aligned}
$$

where $f^{\prime}=\sum_{i} f_{i}^{\prime} \mathbf{e}_{i}$ is defined by

$$
f_{i}^{\prime}=\frac{\sqrt{\mu_{v}}}{\sqrt{\operatorname{deg}\left(v_{i}\right)}} f_{i}
$$

By $\operatorname{deg}(v)=Y_{1, v, n}+Y_{2, v, n}$ and (5-2), we know that, for any $\varepsilon>0$, there is a constant $a$, and

$$
\begin{aligned}
& \mathbb{P}\left(\left|\operatorname{deg}(v)-\mu_{v}\right|>\varepsilon \mu_{v} \text { for some } v \in V\right) \\
< & 2 \# V \times 2 \exp \left(-\frac{\varepsilon^{2} \mu_{v}}{3}\right) \\
= & 4(2 m)^{n} \exp \left(-\frac{\varepsilon^{2} 2 c_{0}(2 m)^{\varepsilon_{0} l-(2 k+1)}}{3}\right) \\
\leq & 2 a \exp \left(-a^{\prime}(2 m)^{\varepsilon_{0} l}\right),
\end{aligned}
$$

where $a, a^{\prime}$ can be taken as the same constants as the preceding subsection. Thus we have, for any given $\varepsilon>0$,

$$
\left\|M^{\prime}\right\| \leq\|B\|(1-\varepsilon)^{-1}
$$

with probability $1-2 a \exp \left(-a^{\prime}(2 m)^{\varepsilon_{0} l}\right)$, which is independent of $c_{0}$ (but depends on $m, k, c$, and $\varepsilon$ ).

On the other hand, since $M=D A D-\pi_{0}$, we see that

$$
M-M^{\prime}=\mu_{e} D K D-\pi_{0} .
$$

With respect to the orthonormal basis $\left\{\mathbf{e}_{i}\right\}, \pi_{0}$ can be expressed as

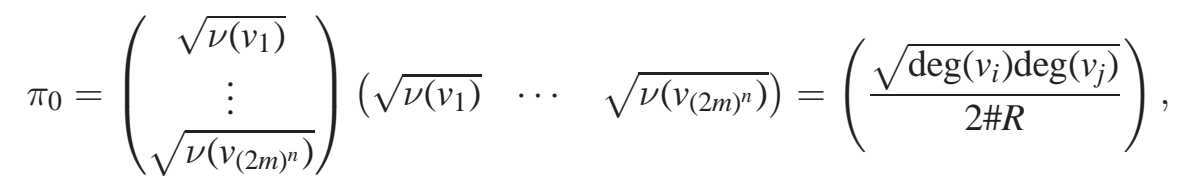


and hence

$$
\begin{aligned}
M-M^{\prime} & =\left(\frac{\mu_{e}}{\sqrt{\operatorname{deg}\left(v_{i}\right) \operatorname{deg}\left(v_{j}\right)}}-\frac{\sqrt{\operatorname{deg}\left(v_{i}\right) \operatorname{deg}\left(v_{j}\right)}}{2 \# R}\right) \\
& =\frac{1}{2 \# R}\left(\frac{2 \# R \mu_{e}-\operatorname{deg}\left(v_{i}\right) \operatorname{deg}\left(v_{j}\right)}{\sqrt{\operatorname{deg}\left(v_{i}\right) \operatorname{deg}\left(v_{j}\right)}}\right) .
\end{aligned}
$$

Therefore we obtain

$$
\begin{aligned}
& \| M-M^{\prime}|| \\
= & \max _{|f|=1}\left|\left(f,\left(M-M^{\prime}\right) f\right)\right| \\
= & \frac{1}{2 \# R} \max _{|f|=1}\left|\sum_{i} f_{i}\left(\sum_{j} \frac{2 \# R \mu_{e}-\operatorname{deg}\left(v_{i}\right) \operatorname{deg}\left(v_{j}\right)}{\sqrt{\operatorname{deg}\left(v_{i}\right) \operatorname{deg}\left(v_{j}\right)}} f_{j}\right)\right| \\
= & \frac{1}{2 \# R} \max _{|f|=1}\left|\sum_{i} \sqrt{\operatorname{deg}\left(v_{i}\right)} f_{i}\left(\sum_{j}\left(\frac{2 \# R \mu_{e}}{\operatorname{deg}\left(v_{i}\right) \operatorname{deg}\left(v_{j}\right)}-1\right) \sqrt{\operatorname{deg}\left(v_{j}\right)} f_{j}\right)\right| .
\end{aligned}
$$

Recall that, by definition, $\# R$ is a binomially distributed random variable with distribution $\operatorname{Bi}\left((2 m)^{\eta},\left(c_{0}(2 m)^{d \eta}\right) /(2 m)^{\eta}\right)$. Thus the expected value of \#R is equal to $c_{0}(2 m)^{d \eta}$. Noting that

$$
2 c_{0}(2 m)^{d \eta} \mu_{e}=2 c_{0}(2 m)^{d \eta} \times 2 c_{0}(2 m)^{d \eta-2 n}=4 c_{0}^{2}(2 m)^{2 d \eta}(2 m)^{-2 n}=\mu_{v}^{2}
$$

and (5-2), we see that, for any $\varepsilon>0$,

$$
\begin{aligned}
\mathbb{P}\left(\left|2 \# R \mu_{e}-\mu_{v}^{2}\right|>\varepsilon \mu_{v}^{2}\right) & =\mathbb{P}\left(\left|\# R-c_{0}(2 m)^{d \eta}\right|>\varepsilon c_{0}(2 m)^{d \eta}\right) \\
& \leq 2 \exp \left(-\frac{\varepsilon^{2} c_{0}(2 m)^{d \eta}}{3}\right) \\
& \leq 2 \exp \left(-\frac{\varepsilon^{2} c_{0}}{3}(2 m)^{k l+\varepsilon_{0} l}\right) \\
& \leq b \exp \left(-b^{\prime}(2 m)^{\varepsilon_{0} l}\right)
\end{aligned}
$$

holds, where

$$
b=2, \quad b^{\prime}=\frac{\varepsilon^{2} c^{-1}}{3} .
$$

Note that we may assume $2 a>b$ and $a^{\prime}<b^{\prime}$, which implies

$$
\mathbb{P}\left(\left|2 \# R \mu_{e}-\mu_{v}^{2}\right|>\varepsilon \mu_{v}^{2}\right)<2 a \exp \left(-a^{\prime}(2 m)^{\varepsilon_{0} l}\right) .
$$


By (6-12) and (6-14), we see that, for any given $\varepsilon>0$,

$$
\begin{aligned}
& \left\|M-M^{\prime}\right\| \\
\leq & \frac{1}{2 \# R} \max _{|f|=1}\left|\sum_{i} \sqrt{\operatorname{deg}\left(v_{i}\right)} f_{i}\left(\left(\frac{1+\varepsilon}{(1-\varepsilon)^{2}}-1\right) \sum_{j} \sqrt{\operatorname{deg}\left(v_{j}\right) f_{j}}\right)\right| \\
\leq & \frac{1}{2 \# R} \max _{|f|=1} \sqrt{\sum_{i} \operatorname{deg}\left(v_{i}\right)}|f| \\
= & \frac{1}{2 \# R}\left(\frac{\varepsilon \varepsilon-\varepsilon^{2}}{1-\varepsilon}+\frac{2 \varepsilon}{(1-\varepsilon)^{2}} \sqrt{\sum_{j} \operatorname{deg}\left(v_{j}\right)|f|}\right) \\
= & \frac{\varepsilon}{1-\varepsilon}+\frac{2 \varepsilon}{(1-\varepsilon)^{2}}
\end{aligned}
$$

with probability $1-4 a \exp \left(-a^{\prime}(2 m)^{\varepsilon_{0} l}\right)$, where Cauchy-Schwarz inequality is used in the second inequality and $\sum_{j} \operatorname{deg}\left(v_{j}\right)=2 \# E_{P}=2 \# R$ implies the last equality. Thus, together with (6-13), for any $\varepsilon>0$, we see that

$$
\|M\| \leq\left\|M^{\prime}\right\|+\left\|M-M^{\prime}\right\| \leq \frac{\|B\|}{1-\varepsilon}+\frac{\varepsilon}{1-\varepsilon}+\frac{2 \varepsilon}{(1-\varepsilon)^{2}}
$$

holds with probability greater than $1-6 a \exp \left(-a^{\prime}(2 m)^{\varepsilon_{0} l}\right)$, which is independent of $c_{0}$ (but depends on $m, k, c$, and $\varepsilon$ ). Recall that (6-11) tells us that we may assume $\|B\|<\varepsilon$ with high probability. By taking $\varepsilon<1 / 2$, we may assume

$$
\frac{2 \varepsilon}{1-\varepsilon}+\frac{2 \varepsilon}{(1-\varepsilon)^{2}}<12 \varepsilon
$$

Thus we obtain, by taking (6-11) in account,

$$
\mathbb{P}(\|M\| \leq 12 \varepsilon) \geq 1-a_{1} \exp \left(-a_{2}(2 m)^{\varepsilon_{0} l}\right)-a_{3}(2 m)^{-\left(\alpha \varepsilon_{0}-k\right) l},
$$

where

$$
a_{1}=6 a, a_{2}=a^{\prime}, a_{3}=\frac{c^{\prime}(\alpha)}{\varepsilon^{2 \alpha}} .
$$

By taking $\varepsilon=\varepsilon^{\prime} / 12$, this completes the proof of (6-9).

\section{Random quotients}

In the proof of Propositions 2.1 and 3.7, we did not use the fact that $\Gamma$ is a free group generated by $S ; \Gamma$ can be any group generated by $S$ (see [18, IV.c] for related comments). Therefore what we have actually proven is the following fixed-point theorem for random quotients: 
Theorem 7.1 Let $\mathcal{H}$ be a Hilbert space. Suppose that $\Gamma$ is a group generated by $S=\left\{s_{1}, \ldots, s_{m}, s_{1}^{-1}, \ldots, s_{m}^{-1}\right\}$, and let $\Gamma_{P}^{\prime}=\Gamma / \bar{R}$ for $P \in P(m, \eta, d)$, where $\bar{R}$ denotes the normal closure of the subset of $\Gamma$ determined by $R$. For $1 / 3<d$,

$$
\lim _{\eta \rightarrow \infty} \frac{\#\left\{P \in P(m, \eta, d) \mid \Gamma_{P}^{\prime} \text { has } F(\mathcal{H})\right\}}{\# P(m, \eta, d)}=1
$$

holds.

Theorem 7.2 Let $Y$ be a CAT(0) space. Suppose that $\Gamma$ is a group generated by $S=\left\{s_{1}, \ldots, s_{m}, s_{1}^{-1}, \ldots, s_{m}^{-1}\right\}$, and let $\Gamma_{P}^{\prime}=\Gamma / \bar{R}$ for $P \in P(m, \eta, d)$, where $\bar{R}$ denotes the normal closure of the subset of $\Gamma$ determined by $R$. Take $0 \leq \delta<1$ and an integer $k$ so that $k>1 /(1-\delta)$. Let $\mathcal{Y}_{\delta}$ be the class of CAT( 0$)$ spaces with $\delta(Y) \leq \delta$. Then, for $k /(2 k+1)<d$,

$$
\lim _{\eta \rightarrow \infty} \frac{\#\left\{P \in P(m, \eta, d) \mid \Gamma_{P}^{\prime} \text { has } F(Y) \text { for all } Y \in \mathcal{Y}_{\delta}\right\}}{\# P(m, \eta, d)}=1
$$

holds.

In the theorems above, we cannot say $\Gamma$ is infinite in general. What is known in this direction is the following theorem for hyperbolic groups due to Ollivier:

Theorem 7.3 ([19, Theorem 4]) Let $\Gamma$ be a hyperbolic group with harmless torsion generated by $S=\left\{s_{1}, \ldots, s_{m}, s_{1}^{-1}, \ldots, s_{m}^{-1}\right\}$ and $\theta$ the gross cogrowth of $\Gamma$. Suppose that $d<1-\theta$. Then we have

$$
\lim _{\eta \rightarrow \infty} \frac{\#\left\{P \in P(m, \eta, d) \mid \Gamma_{P}^{\prime} \text { is non-elementary hyperbolic }\right\}}{\# P(m, \eta, d)}=1 .
$$

See [19] for the definitions of gross cogrowth and harmless torsion. We remark here that the gross cogrowth of the free group of rank $m$ is equal to $\frac{1}{2} \log _{2 m}(8 m-4)$.

\section{References}

[1] B. Bekka, P. de la Harpe, and A. Valette, Kazhdan's Property (T), New Mathematical Monograph 11, Cambridge Univ. Press, 2008.

[2] B. Bollobás, Random Graphs, Second Edition, Cambridge Studies in Advanced Mathematics 73, Cambridge University Press, 2001.

[3] M. R. Bridson and A. Haefliger, Metric Spaces of Non-Positive Curvature, SpringerVerlag, Berlin, Heidelberg, 1999. 
[4] F. Chung, L. Lu, and V. Vu, The spectra of random graphs with given expected degrees, Internet Math., 1 (2003), 257-275.

[5] D. Fisher and L. Silberman, Groups not acting on manifolds, Int. Math. Res. Not. IMRN 2008, 16, Art. ID rnn060, 11 pp.

[6] K. Fujiwara and T. Toyoda, Random groups have fixed points on $C A T(0)$ cube complexes, Proc. Amer. Math. Soc. 140 (2012), 1023-1031.

[7] Z. Füredi and J. Komlós, The eigenvalues of random symmetric matrices, Combinatorica 1 (1981), 233-241.

[8] M. Gromov, Random walk in random groups. GAFA 13 (2003), 73-148.

[9] H. Izeki, T. Kondo, and S. Nayatani, Fixed-point property of random groups, Annals of Global Analysis and Geom. 35 (2009), 363-379.

[10] H. Izeki, T. Kondo, and S. Nayatani, N-step energy of maps and fixed-point property of random groups, to appear in Groups, Geometry, and Dynamics.

[11] H. Izeki and S. Nayatani, Combinatorial harmonic maps and discrete-group actions on Hadamard spaces, Geom. Dedicata 114 (2005), 147-188.

[12] S. Janson, T. Łuczak, and A. Ruciński, Random Graphs, Wiley, 2000.

[13] T. Kondo, Fixed-point property for CAT(0) spaces, preprint.

[14] T. Kondo, CAT(0) spaces and expanders, to appear in Math. Z.

[15] M. Korevaar and R. Schoen, Sobolev spaces and harmonic maps for metric space targets, Comm. Anal. Geom. 1 (1993), 561-659.

[16] M. Kotowski and M. Kotowski, Random groups and property $(T)$ : Zuk's theorem revisited, J. London Math. Soc., 88 (2013), 396-416.

[17] A. Naor and L. Silberman, Poincare inequalities, embeddings, and wild groups, Compositio Mathematica 147 (2011), 1546-1572

[18] Y. Ollivier, A January 2005 invitation to random groups, Ensaios Mathemáticos [Mathematical Surveys], 10, Sociedade Brasileira de Mathemática, Rio de Janeiro, 2005.

[19] Y. Ollivier, Sharp phase transition theorems for hyperbolicity of random groups, GAFA 14 (2004), 595-679.

[20] J.-P. Serre, Trees, Translated by J. Stillwell, Springer-Verlag, Berlin, Heidelberg, New York, 2000.

[21] L. Silberman, Addendum to "Random walk on random groups" by M. Gromov. GAFA 13 (2003), 147-177.

[22] M.-T. Wang, Generalized harmonic maps and representations of discrete groups, Comm. Anal. Geom. 8 (2000), 545-563.

[23] A. Zuk, Property (T) and Kazhdan constants for discrete groups, GAFA 13 (2003), 643-670. 
Department of Mathematics, Keio University, 3-14-1 Hiyoshi, Kohoku-ku, Yokohama, 2238522, Japan

izeki@math.keio.ac.jp 